\title{
The atmospheric circulation over the North Atlantic as induced by the SST field
}

\author{
Yizhak Feliks $^{1}$, Michael Ghil ${ }^{2,3}$ and Andrew W. Robertson ${ }^{4}$ \\ Dept. of Atmospheric \& Oceanic Sciences and Institute of Geophysics \& Planetary Physics, \\ University of California, Los Angeles, USA
}

August 20, 2010

${ }^{1}$ Permanent address: Department of Mathematics, Israel Institute of Biological Research, Ness Ziona, Israel

2 Additional affiliation: Geosciences Department and Laboratoire de Météorologie Dynamique (CNRS and IPSL), Ecole Normale Supérieure, Paris, France.

${ }^{3}$ Corresponding author: Geosciences Department and LMD, Ecole Normale Supérieure, 24 rue Lhomond, F-75321 Paris Cedex 05, France

E-mail: ghil@atmos.ucla.edu.

${ }^{4}$ Permanent address: International Research Institute for climate and society (IRI), Monell Building, Room 230, P. O. Box 1000, Palisades, NY 10964-8000, USA 


\section{ABSTRACT}

Spectral analyses of the sea surface temperature (SST) in the Simple Ocean Data Analysis (SODA) reanalysis for the past half-century identify prominent and statistically significant interannual oscillations in two regions along the Gulf Stream front over the North Atlantic. A model of the atmospheric marine boundary layer coupled to a baroclinic quasi-geostrophic model of the free atmosphere is then forced with the SST history from the SODA reanalysis. Two extreme states are found in the atmospheric simulations: they consist of (1) an eastward extension of the westerly jet associated with the front, which occurs mainly during boreal winter; and (2) a quiescent state of very weak flow found predominantly in the summer. This vacillation of the oceanic-front-induced jet in the model is found to exhibit periodicities similar to those identified in the observed Gulf Stream SST front itself. In addition, a close correspondence is found between interannual spectral peaks in the observed North Atlantic Oscillation (NAO) index, and the SODA-induced oscillations in the atmospheric model. In particular, significant oscillatory modes with periods of $8.5,4.2$ and 2.8 years are found in both the observed and simulated indices, and shown to be highly synchronized and of similar energy in both time series. These oscillatory modes in the simulations are shown to be suppressed when either (a) the Gulf Stream front or (b) its interannual oscillations are omitted from the SST field. Moreover, these modes also disappear when (c) the SST front is spatially smoothed, thus confirming that they are indeed induced by the oceanic front. 


\section{Introduction}

Interannual and interdecadal oscillations emerge above a red-noise spectrum in many instrumental and proxy records of climate from the North Atlantic and the continents bordering it; see for example Bjerknes (1964), Deser and Blackmon (1993), Dettinger et al. (1995), Plaut et al. (1995), Hurrell and van Loon (1997), Moron et al. (1998), Da Costa et al., (2002), Kushnir et al. (2002), Hurrell et al. (2003), Feliks et al. (2010a), and many others. The extent to which these oscillations may be ocean forced is still controversial, first because of the lack of consensus in atmospheric general circulation model (GCM) studies with prescribed sea surface temperatures (SSTs; e.g., Robertson et al. 2000; Kushnir et al. 2002; Manganello 2008), and second due to the substantial influence that mid-latitude atmospheric perturbations exert on the underlying ocean (Frankignoul and Hasselman 1977).

More recently, Feliks et al. $(2004,2007)$ have presented modeling evidence that sharp SST gradients associated with mid-latitude ocean fronts, such as the Gulf Stream and the Kuroshio Extension, can exert a strong influence on the overlying atmosphere, as well as on its low-frequency variability. In particular, this type of SST front was shown to force an atmospheric jet above it that extends vertically through the troposphere, and the variations in frontal strength were shown to modulate the atmospheric jet's intensity. Since oceanic fronts have a width of about $100 \mathrm{~km}$ or less, they are not resolved by conventional GCMs, which typically have grid sizes of $200 \mathrm{~km}$ or more. However, recent GCM simulations with a resolution of roughly $50 \mathrm{~km}$ by Minobe et al. (2008) have confirmed the findings of Feliks et al. $(2004,2007)$ in terms of the existence of the atmospheric jet. Still, such GCMs cannot yet be run for the extended times needed to ascertain the impact of the SST fronts on the atmospheric jet's interannual and interdecadal variability. 
In a spectral analysis of several climate indices, Feliks et al. (2010a) found a prominent oscillatory mode with a 7-8-yr period in indices over the North Atlantic, the Eastern Mediterranean, and the Ethiopian Plateau. An energy analysis of the synchronization between the projections of this oscillatory mode on the different regions analyzed suggested that the 7 8-yr mode might originate in the North Atlantic Ocean. Indeed, an oscillation with this period has been reported in several other studies of North Atlantic climate (Rogers 1984; Hurrel and Loon 1997; Robertson 2001; Da Costa and de Verdière 2002; Gámiz-Fortis et al. 2002; Paluš and Novotná 2004). Feliks et al. (2010a) also detected an oscillatory mode with an approximate 4-yr period in all of the above regions; this mode appeared again to be associated with the North Atlantic, as well as to the quasi-quadrennial oscillation in the El Niño-Southern Oscillation (ENSO) variability.

The purpose of this paper is to show that the high-resolution SST history for the 50-yr interval 1958-2007, produced by the Simple Ocean Data Assimilation (SODA) reanalysis (Carton and Giese 2008), (i) exhibits interannual to near-decadal oscillations in the Gulf Stream's frontal region, and (ii) that these oscillations are capable of influencing the atmosphere above it, as simulated by a model of intermediate complexity. A brief introduction to the SODA data and to our atmospheric model is given in section 2; the model consists of a marine atmospheric boundary layer (MABL) coupled to a baroclinic, quasi-geostrophic (QG) model of the free atmosphere. We then analyze the SODA SST data in section 3 and the model's response to it in section 4, using multichannel singular spectrum analysis (MSSA) to identify interannual oscillations in each dataset. In section 5, we compare the observed NAO index and the simulated index. In section 6 , we summarize the results. 


\section{Data and Methods}

\section{a. The Simple Ocean Data Assimilation (SODA) dataset}

The SODA reanalysis of ocean climate variability from Carton and Giese (2008), version 2.0.24, provides the monthly SST data set used in this study over the 50-yr interval 1958-2007, with a grid resolution of 0.5 degrees. The $\mathrm{SST}$ is taken to equal the temperature in the upper $5 \mathrm{~m}$ of the ocean. We also use the SODA sea surface height $(\mathrm{SSH})$ field, which corresponds to the subsurface velocity, to aid us in the interpretation of the oscillations found in the SST field. The SODA is based on assimilating global-ocean observations into an ocean GCM, originally developed at NOAA's Geophysical Fluid Dynamics Laboratory at Princeton and using the Parallel Ocean Program numerics (Smith et al. 1992); the observations include temperature and salinity profiles, additional hydrography, SSTs, and altimeter sea level data.

The annual mean SST field from SODA over the North Atlantic is shown in Fig. 1. The Gulf Stream front in SST is very prominent, with temperature changes of $7 \mathrm{~K} / 100 \mathrm{~km}$ across the front. The front is aligned with the Florida Current, which flows along the east coast of North America until the separation point at Cape Hatteras. A strong thermal front then extends into the open ocean until $40 \mathrm{~W}$, beyond which it weakens abruptly and the isotherms diverge. The mean SSH field over the same area is shown in Fig. 2. The Gulf Stream front in SSH follows closely the SST front, with cross-frontal gradients reaching $0.5 \mathrm{~m} / 100 \mathrm{~km}$; this gradient corresponds to a subsurface current speed of about $0.5 \mathrm{~ms}^{-1}$. 


\section{b. The atmospheric model}

The atmospheric model has a marine atmospheric boundary layer (MABL), coupled to a quasigeostrophic (QG) baroclinic model of the free atmosphere. The MABL and QG models are described in detail by Feliks et al. (2004) and Feliks et al. (2007), respectively; these papers are referred to as FGS04 and FGS07 in the following. For completeness, we provide here a summary of the main aspects of the model.

The frictional MABL model assumes a well-mixed moist boundary layer, in equilibrium with the underlying SST field $T=T(x, y)-$ i.e., the virtual potential temperature within the MABL is constant - while the flow in the free atmosphere above it is assumed to be geostrophic. The pressure gradient in the MABL has two components: one is due to the geostrophic wind in the free atmosphere at the top of the MABL and is independent of $z$; this is the component familiar from boundary layer theory in all areas of geophysical fluid dynamics. The second component is due to the underlying thermal front and it is a function of $z$.

The linear equations of motion in the MABL are:

$$
\begin{aligned}
& k \frac{\partial^{2} w}{\partial z^{x}}+f v-\frac{1}{\rho} \frac{\partial p}{\partial x}=0, \\
& k \frac{\partial^{2} w}{\partial z^{x}}-f u-\frac{1}{\rho} \frac{\partial p}{\partial y}=0,
\end{aligned}
$$

while the boundary conditions are:

$$
\begin{gathered}
u=v=0 \quad \text { at } \quad z=0, \\
u=u_{G}, v=v_{G} \quad \text { as } \quad z \rightarrow \infty .
\end{gathered}
$$


The vertical wind profiles within the MABL can then be derived analytically in closed form, yielding the vertical velocity $w\left(H_{E}\right)$ at the top of the MABL by integrating the continuity equation:

$$
w\left(H_{E}\right)=\frac{H_{E}}{2 \pi}\left[\zeta_{G}-\frac{g H_{E}}{f \theta_{0}}\left(1-\frac{1}{2 \pi}\right) \nabla^{2} \mathrm{~T}\right] \quad .
$$

Here $\zeta_{G}=\nabla^{2} \psi$ is the geostrophic vorticity at the top of the MABL, while $\psi$ is the geostrophic streamfunction. The MABL depth, $H_{E}=\pi \sqrt{2 k_{0} / f^{2}}$, depends on latitude through the Coriolis parameter $f$, and $k_{0}$ is a turbulent eddy coefficient. Numerical values of model parameters for the simulations of Sec. 4 are given in Table 1.

The vertical velocity in (3) has two components: the first is associated with the geostrophic flow above the MABL, and we call it the mechanical component; the second is associated with the Laplacian $\nabla^{2} T$ of the SST and we refer to it as the thermal component. The conventional Ekman pumping velocity given by the mechanical component is thus augmented here by a thermal component driven by $\nabla^{2} T$. Note that while $\zeta_{g}$ above the MABL is ultimately driven by $\nabla^{2} T$, since no other thermal forcing is included in the model, it is also a function of the dynamics of the free atmosphere, modeled here by using a QG baroclinic model. For our purposes, the analytical MABL model of FGS04 has advantages over the Lindzen and Nigam (1987) model, which was developed for the tropics, because ours is directly coupled to the free atmosphere's QG dynamics, and the latter plays an important role in mid-latitudes.

The baroclinic model of FGS07 consists of the standard QG potential vorticity equation (Pedlosky 1987; Holton 1992), with vertical decomposition in terms of the barotropic and first baroclinic normal modes following Flierl (1978) and Feliks and Ghil (1996). The equation for the modal amplitudes (Flierl 1978) is: 


$$
\frac{\partial q_{k}}{\partial t}+\beta \frac{\partial \psi_{k}}{\partial x}+\sum_{k} \xi_{i j k} J\left(\psi_{i} q_{j}\right)=r_{H} \nabla^{4} \psi_{k-} \frac{H}{H_{a}} \phi_{k}(0) w_{a}(z=0)
$$

where we only retain $k=0,1$ and

$$
q_{k}=\nabla^{2} \psi_{k}-\lambda_{k}^{2} \psi_{k}, \xi_{i j k}=\int \phi_{i} \phi_{j} \phi_{k} d z
$$

The triple interaction coefficients $\xi_{i j k}$ are all equal to zero or one except $\xi_{111}$; we shall refer to the value of $\xi_{111 \text { by }} \xi$.

Using (1) for $w\left(H_{E}\right)$, the lower boundary condition for the free atmosphere at $z=0$ is

$$
\frac{H}{H_{a}} \frac{d}{d t}\left[\frac{\partial}{\partial z}\left(\frac{1}{s} \frac{\partial \psi}{\partial z}\right)\right]=\frac{H}{H_{a}} w_{a}(x, y, z=0, t)=\gamma \nabla^{2} \psi-\alpha \nabla^{2} T
$$

here $\alpha=\frac{1}{2 \pi}\left(1-\frac{1}{2 \pi}\right) \frac{g H_{E}^{2}}{H_{a} V^{2}} \frac{T^{*}}{\theta_{0}}=\frac{1}{2 \pi}\left(1-\frac{1}{2 \pi}\right) \frac{H_{E}}{H_{a}} \frac{1}{F_{r}^{2}}, \gamma=\frac{1}{2 \pi} \frac{H_{E}}{H_{a}} \frac{f L}{V}=\frac{1}{2 \pi \varepsilon} \frac{H_{E}}{H_{a}}$,

$H_{a}$ is the height of the free atmosphere, $L$ is the horizontal length scale across the front, $V$ scales the wind speed in the cross-front direction, and the (nondimensional) temperature $T$ scales like the oceanic frontal strength $T^{*}$. The constants $\alpha$ and $\gamma$ determine the relative importance of the coupling between the MABL and QG dynamics above. Note that $\alpha \sim 1 / F r^{2}$ and $\gamma \sim 1 / \varepsilon$, and that both are proportional to $H_{E} / H_{a}$, where $F r$ is the Froude number, $\varepsilon$ the Rossby number, and $H_{E} / H_{a}$ is the ratio between the height of the MABL and that of the free atmosphere. Since $\alpha$ is proportional to the strength $T^{*}$ of the SST front, it is clear that stronger SST fronts induce stronger atmospheric circulations. A thicker MABL has a larger influence on the spin- up by the SST front than on the spin-down by the mechanical component, since $\alpha \sim H_{E}{ }^{2}$, while $\gamma \sim H_{E}$.

The physical mechanism represented by the coupled MABL-QG model, by which the SST front influences the free atmosphere, is sketched in Fig. 3a. Two mechanisms combine to spin up the strong eastward jet. The first mechanism is based on a mid-latitude stirring — from 
initial zero momentum - and it induces a strong, narrow eastward jet at the latitude of the stirring, and a weak broad westward flow at the other latitudes, due to the $\beta$ effect. The strong jet is the result of keeping the net momentum zero (Salmon 1998, p. 277, Fig. 6.4), and its length is determined by the Rhines scale. This mechanism is prominent in the upper layers, as shown in Fig. 12 of section 4a, at an altitude of $10 \mathrm{~km}$.

The second mechanism is a "breeze-like" circulation induced by the sharp SST gradient across the front: this SST gradient causes a pressure gradient in the MABL that gives rise to a transverse ageostrophic circulation; the latter leads, via mass conservation, to a thermally induced pumping of the free atmosphere. This pumping then spins up a jet that runs parallel to the front, extends through the depth of the free atmosphere, and is governed by QG dynamics, as shown by Feliks et al. (2010b). This mechanism is relevant to the lower layers, since the breeze-like circulation is found below $3 \mathrm{~km}$, as seen in the surface layer of Fig. 12. As the SST front weakens, the thermal component of $w\left(H_{E}\right)$ decreases and the mechanical components tends to spin the jet down. So we can expect the length of the jet in the upper layers to differ from that in the lower layers; our model simulation of section 4a confirms this difference, and it appears indeed in Fig. 12.

No large-scale temperature gradients are imposed in the QG model, which thus represents only the atmospheric response to the SST-induced vertical velocity, rather than to equator-pole temperature differences. Nonetheless, this jet is in thermal wind balance and is both barotropically and baroclinically unstable, as discussed in FGS07. Snapshots of the thermal and mechanical components of $w\left(H_{E}\right)$ are shown in Fig. 3b. The thermal component is larger by two orders of magnitude than the mechanical component above the oceanic front and so it gives rise to the strong atmospheric jet; see Fig. 12 below. In both components the large 
values follow closely the Gulf Stream front between Cape Hatteras and $42 \mathrm{~W}$; farther to the east, the SST front weakens abruptly and so do both components of $w\left(H_{E}\right)$. The small-scale structures found in the mechanical component are due to the tendency of the enstrophy, $\left(\nabla^{2} \psi\right)^{2}$, to cascade to the smaller scale in QG turbulence (Charney 1971).

The correspondence between two-mode and two-level models (Flierl 1978) is given by

$$
H_{1}=\frac{\left(\sqrt{\xi^{2}+4}-\xi\right)^{2}}{4+\left(\sqrt{\xi^{2}+4}-\xi\right)^{2}}
$$

where $H_{1}$ is the nondimensional upper-layer depth and $H_{2}=1-H_{1}$ is the lower-layer depth. For an interaction coefficient $\xi=0$ we have $H_{1}=H_{2}=1 / 2$, whereas large negative values of $\xi$ give $H_{2} \sim \xi^{2}$. In the basic experiment of section 4 we take $\xi=-1$, which corresponds to the dimensional values $H_{1} H=7230 \mathrm{~m}$ and $H_{2} H=2770 \mathrm{~m}$, given $H=10000 \mathrm{~m}$ (see Table 1); values of $\xi=-1$ or $H_{2}: H_{1}=3 / 7$ are often used in atmospheric models (e.g., Kravtsov et al. 2005).

The streamfunction at any height $z$ is given by

$$
\psi(z)=\psi_{B T}+\emptyset_{1}(z) \psi_{B C}
$$

where $\phi_{1}(z)$ is the vertical structure of the first baroclinic mode, $\phi_{1}(0)=2$ corresponds to the surface $(z=0)$, i.e. at the top of the MABL, and $\phi_{1}(H)=-2$ corresponds to the top of the free atmosphere, i.e. at an altitude of $10 \mathrm{~km}$.

Along the meridional boundaries, we impose free-slip conditions, $\psi=q=0$, while in the zonal direction we use periodic boundary conditions with sponge layers in the $\Delta L=1000$ $\mathrm{km}$ adjacent to the boundaries. To examine the role of the width $\Delta L$ of the sponge layer on the 
solution, we carried out two additional experiments with different widths, $\Delta L=750 \mathrm{~km}$ and $\Delta L$ $=500 \mathrm{~km}$; see section $4 \mathrm{~b}$.

The numerical scheme is the same as in Feliks (1990) and in Feliks and Ghil (1996). Key features include fourth-order accurate discretization by finite elements in the horizontal (cf. Haidvogel et al. 1980) and a second order Adams-Bashforth scheme in time. FGS04 showed that the correct generation of an atmospheric jet by the SST front requires a horizontal grid resolution of $50 \mathrm{~km}$ or less, as confirmed by Minobe et al. (2008). At slightly lower resolution, FGS04 also indicated how to increase the nominal strength of the oceanic front in a self-consistent way in order to compensate for the loss of adequate resolution. FGS07 also studied the role of the two layers' depth ratio $H_{2}: H_{1}$ on the evolution. They found that the baroclinic transients became more and more significant, though, as this ratio tends to 1 , and hence all longer-term behavior, on the time scale of months, is mostly baroclinic.

\section{c. Spectral analysis methods}

The primary methods of spectral analysis used here to identify oscillatory modes of variability in the SODA data and atmospheric model simulations are univariate singular spectral analysis (SSA: Broomhead and King 1986a; Vautard and Ghil, 1989; Ghil et al. 2002a) and multichannel SSA (MSSA). MSSA is an extension of SSA to several time series, with each "channel" corresponding to one of the scalar time series of interest (Broomhead and King 1986b; Keppenne and Ghil 1993; Plaut and Vautard 1994; Ghil et al. 2002a). The method essentially diagonalizes the lag-covariance matrix associated with the vector time series whose components are the channels. The eigenvectors of this matrix are the space-time empirical 
orthogonal functions (ST-EOFs) and the projection of the original data onto them yields the corresponding principal components (PCs).

MSSA is therewith a data-adaptive method of spectral analysis, whereby oscillatory components of variability — that may be modulated in phase and amplitude over time — are captured as pairs of eigenmodes with similar eigenvalues. A Monte-Carlo statistical significance test against red noise is performed by comparing the projection of the data onto a given ST-EOF for each channel with the corresponding projections of a large ensemble of rednoise surrogates. The power spectrum of this Monte-Carlo SSA (MC-MSSA; Allen and Robertson, 1996; Ghil et al., 2002a) method consists of an eigenvalue spectrum plotted against the dominant frequency associated with each eigenvalue $k$, estimated from the reduced Fourier transform of the associated PC timeseries (Vautard et al. 1992). The entire vector time series or parts thereof that correspond to trends, oscillatory modes or noise can be reconstructed by using linear combinations of these PCs and ST-EOFs, yielding spatio-temporal reconstructed components (RCs).

\section{Spectral analysis of SODA data}

In order to examine the impact of the Gulf Stream front on the atmosphere we first analyze the frequency spectra of SST and SSH along the Gulf Stream track, performing the analysis in two rectangular regions where the interannual oscillations of $\nabla^{2} T$ exhibit their largest values. These two regions correspond to (1) the Cape Hatteras region (CHR; $34 \mathrm{~N}-43.50 \mathrm{~N}, 75 \mathrm{~W}-60 \mathrm{~W}$ ), where the Gulf Stream front associated with the subtropical gyre separates from the coast; and (2) the Grand Banks region (GBR; $42 \mathrm{~N}-50 \mathrm{~N}, 55 \mathrm{~W}-35 \mathrm{~W})$, where the Gulf Stream front 
diverges abruptly, due to the confluence between the subtropical and subpolar gyres. The two regions are outlined in Fig. 1.

We first apply MSSA to the SST data over the entire North Atlantic domain with a window width of $M=150$ months. The leading pair of ST-EOFs 1 and 2 captures the strongest oscillatory mode; it has a period of $1 \mathrm{yr}$, contains $90 \%$ of the variance, and represents the very strong seasonal cycle in SSTs. Phase composites of the corresponding reconstructed components, denoted by $\operatorname{RCs}(1,2)$, are shown in Fig. 4, where the cycle has been divided into eight phase categories, using the methodology of Moron et al. (1998).

The seasonal changes are strongest to north of the front, with a peak amplitude of almost $20 \mathrm{~K}$. The seasonal movement of the SST front is most prominent between $35 \mathrm{~N}$ and 40 $\mathrm{N}$ along the coast and on the continental shelf downstream of Cape Hatteras; the separation point of the front moves quite visibly northward during the summer. The SST gradient across the front decreases during the summer and increases during the winter. Thus we expect a strong atmospheric jet during the winter and weak flow during the summer, as will be seen in Fig. 12.

To isolate interannual oscillations in the CHR and GBR regions, we first filtered out the annual cycle and subannual variability by applying a 12-month running average to the data set; doing so is preferable to the simple use of annual means, cf. Feliks et al. (2010a). Next, we applied MSSA to the pre-filtered data in each region separately, with a window width of $M=$ 150 months. Only those signals that are significant at the (two-sided) $95 \%$ level will be analyzed further below.

The MSSA spectrum of the field SST in CHR is shown in Fig. 5, together with error bars that indicate the $95 \%$ confidence interval for a red-noise process. Oscillations with periods 
of about 8.5, 5.2 and 3.8 yr emerge as statistically significant, and will be analyzed below. The leading oscillatory mode, captured by ST-EOFs $(3,4)$, has a period of about $8.5 \mathrm{yr}$ and contains $13 \%$ of the interannual variance. The sum of the two RCs of this oscillatory mode, $\mathrm{RC}(3,4)$, is shown in Fig. 6 at the point $(74 \mathrm{~W}, 37 \mathrm{~N})$, where it reaches its maximum amplitude. The 8.5-yr oscillation (dashed curve) captures quite well the interannual variability of the raw data (solid curve); its amplitude has increased markedly after about 1975.

Phase composites of this leading interannual mode are shown in Fig. 7, again by dividing the cycle into eight phase categories and plotting the first four. The mode consists of SST anomalies elongated along the axis of the mean Gulf Stream front (last panel). These features appear first just off the separation point at Cape Hatteras (first panel), and develop into a meander of the front with maximum amplitudes near $73 \mathrm{~W}$ and $66 \mathrm{~W}$.

The spatial patterns of the phase composites for the 5.2- and 3.8-yr oscillatory modes (not shown) are similar to those in Fig. 7. This similarity suggests the existence of a dominant spatial mode of variability of the front that tends to oscillate at discrete periods. The significant ST-EOFs, ordered by decreasing variance, along with the associated periods and variances, are the following: ST-EOF 5 has a period of $6.2 \mathrm{yr}$ and a variance of 4.3\%; ST-EOF pair $(6,7)$ has a period of $4.5 \mathrm{yr}$ and a variance of $6 \%$, but its amplitude increases by a factor of 2 after 1987 (not shown); and, finally, pair $(8,9)$ has a period of $3.7 \mathrm{yr}$ and a variance of $5 \%$.

In order to test the physical robustness of these interannual modes identified in the SST field, the analysis was repeated using the SSH field within the CHR region, using the same 12month running mean pre-filtering. The leading oscillatory mode again has a period of $8.5 \mathrm{yr}$ and emerges in the ST-EOF $(3,4)$ pair, which accounts for $12 \%$ of the interannual variance. The 
mode's spatio-temporal pattern, shown in Fig. 8 in terms of phase composites, approximately mirrors that seen in the SST field illustrated in Fig. 7.

The spatio-temporal pattern of the 8.5-yr mode in SSTs and SSHs shares certain features with the so-called gyre mode that characterizes the interannual variability in the double-gyre problem of the mid-latitude wind-driven ocean circulation (Jiang et al. 1995; Dijkstra and Ghil 2005). This gyre mode was found to have a dominant $7-8$-yr peak across a hierarchy of ocean models, QG and shallow-water, barotropic and baroclinic (Speich et al. 1995; Ghil et al. 2002b; Simonnet and Dijkstra 2002; Simonnet et al. 2003a, b, 2005; Dijkstra and Ghil 2005). In particular, the gyre mode is known to propagate perpendicular to the direction of the midlatitude jet.

This propagation is quite obvious in the SST anomalies of Fig. 7: in the four composite phases labeled $1.1 \mathrm{yr}$ to $4.3 \mathrm{yr}$, the anomaly propagates from $36-38 \mathrm{~N}$ to $38-40 \mathrm{~N}$, i.e. it propagates in 3 years by about $150 \mathrm{~km}$; this corresponds to a mean propagation speed of $50 \mathrm{~km} / \mathrm{yr}$. In the SSH anomalies of Fig. 8, close examination of the four panels labeled $1.1 \mathrm{yr}$ to 4.3 yr shows that - while there is no propagation of the anomaly features in their entirety the northward expansion of the eddies might still be interpreted as northward propagation of the mean location of the features.

The other significant oscillatory modes in the SSH field also coincide roughly with those found in the SST, as follows: ST-EOF 5 has a period of $6.6 \mathrm{yr}$ and a variance of $4.2 \%$; the triad $(6,7,8)$ has a period of $3.7 \mathrm{yr}$ and variance of $9 \%$; and ST-EOF 9 has a period of $4.2 \mathrm{yr}$ and variance of $2.7 \%$. The phase composites of these significant oscillatory modes again have spatio-temporal patterns similar to those of the 8.5-yr mode. 
To analyze the SST evolution in the GBR rectangle ( $42 \mathrm{~N}-50 \mathrm{~N}, 55 \mathrm{~W}-35 \mathrm{~W})$, we first removed the long-term trend, as captured by RCs $(1,2)$ of the MSS A analysis with a 150 -month window; while the trend was not important in the CHR region, and so it did not need to be subtracted from the raw data there, it accounts here for $18 \%$ of the total variance. After removing it, we applied M-SSA again to the detrended time series with $M=150$ months.

The MC-MSSA spectrum is shown in Fig. 9. The leading oscillatory mode is captured by ST-EOFs $(1,2)$, has a period of $10.5 \mathrm{yr}$, and accounts for $10 \%$ of the total variance of the series, including the trend. This oscillatory mode is shown in Fig. 10 at the point $(47 \mathrm{~W}, 44 \mathrm{~N})$, where it reaches its maximum amplitude within the GBR region.

Phase composites of the 10.5-yr mode in eight phases are shown in Fig. 11. The anomalies are most prominent just eastward of the point where the mean SST front starts to diverge, near 50W; they are broader-scale and noisier than in the CHR region. The oscillatory mode's spatio-temporal evolution suggests an extension-and-contraction of the subpolar gyre.

The other significant oscillatory modes in the SST field are the following: the pair $(3,4)$ has a period of $5.7 \mathrm{yr}$ and captures a variance of $8 \%$, while the pair $(6,7)$ has a period of $3.2 \mathrm{yr}$ and a variance of 5\%. Taken together, these three modes - 10.5, 5.7 and $3.2 \mathrm{yr}$ - account for $23 \%$ of the total variance of the time series, including the trend. The phase composites of these significant oscillatory modes resemble those of the 10.5-yr oscillatory mode in Fig. 11. It seems therefore that, as in the CHR region, a single spatio-temporal mode dominates the interannual variability, but with periods that differ some what from those seen in the other region.

Several differences between the flow of the Gulf Stream in the CHR and GBR regions are described below: they are largely due to the very differe nt nature of the bottom topography and have been investigated over the years in some detail. The North Atlantic's western 
boundary current, after leaving the Florida Straits, flows over the shallow continental shelf, where water depth does not exceed $1000 \mathrm{~m}$, and separates from the East Coast of the United States at Cape Hatteras by crossing the continental rise, where the bottom topography drops sharply to $5000 \mathrm{~m}$ within a few hundred kilometers (Özgökmen et al. 1997).

As the Gulf Stream approaches the Grand Banks, the mass transport by the Gulf Stream decreases, as some of the water is recirculated to the west, some of it continues to the east and crosses the Mid-Atlantic Ridge in the Azores Current, and the remainder forms the North Atlantic Current (NAC) that continues as a well-defined boundary current along the eastern slope of the Grand Banks. At about $51^{\circ} \mathrm{N}$ the NAC moves to the east. As the waters flow eastward, the NAC looses its structure as a well-defined jet, and the water is transported eastward in the Sub-Polar Front, which is the boundary between the warm water in the subtropical gyre and the cooler and less saline water in the subpolar gyre to the north (Rossby, 1999).

These very different bathymetric and water-mass settings can help explain the slight differences between the oscillatory features illustrated in Figs. 5-8 for the CHR and Figs. 9-11 for the GBR. A more detailed study of the interannual variability in the two regions has to be left for subsequent work.

\section{Atmospheric model simulations}

\section{a. Instantaneous and mean fields}

The coupled MABL-QG atmospheric model of Eq. (4) was integrated for $50 \mathrm{yr}$, beginning in January 1958. The only forcing of the model is the monthly mean of $\nabla^{2} T$ as obtained from the SODA data. The initial streamfunction and vorticity were set to zero, and the spin-up of the atmospheric circulation takes less than two months; see FGS07. 
Daily snapshots are shown in Fig. 12 for the evolution of the streamfunction field: barotropic, baroclinic, near the surface and at an altitude of $10 \mathrm{~km}$, respectively. The barotropic streamfunction (upper row of three panels) exhibits intervals during which the jet is both strong and long, such as at time $=1996.3$; this occurs mainly during the winter when the SST front reaches its maximum strength. In this configuration, the simulated atmospheric jet has large meanders that are accompanied by cyclonic flow to the north and anticyclonic flow to the south. The length of the jet is more than $5000 \mathrm{~km}$ and its speed reaches values of $7 \mathrm{~ms}^{-1}$.

There are also time intervals during which the jet practically disappears, such as at time 1993.8; this occurs mainly during the summer, when the SST front reaches its minimum strength. Transitions between the two extreme states are illustrated here at time $=2007.3$. Snapshots of the baroclinic streamfunction taken at the same times (second row in Fig. 12) show that there are intervals during which strong baroclinic eddies accompany the strong jet, such as at time $=1996.3$, while the weak-jet intervals tend to be accompanied by very weak eddy activity, such as at time $=1993.8$. These atmospheric circulation patterns show similarities with those found in FGS04 and FGS07 when using idealized SST fronts. The zonal wavelength of the baroclinic eddies in Fig. 12 is about $20^{\circ}$ longitude or a zonal wavenumber of 18.

The time-mean barotropic and baroclinic streamfunctions fields calculated over the full $50 \mathrm{yr}$ of the simulation are shown in the last panels of Figs. 14 and 15, respectively, in section $4 \mathrm{c}$ below. The mean barotropic jet is strongest between $60 \mathrm{~W}$ and $40 \mathrm{~W}$ and tends to coincide with the GBR region in which the SST front diverges.

Daily snapshots of the surface streamfunction evolution are shown in the third row of Fig. 12. There is a jet roughly between $70 \mathrm{~W}$ and $40 \mathrm{~W}$, just over the Gulf Stream front, and a cyclonic eddy in the western part of the domain. The surface jet has its episodes of weak and 
short jet as at time $=1993.8$ and of strongest and longest jet at time $=1996.3$, like the barotropic mode. The length of the surface jet reaches $3000 \mathrm{~km}$, much shorter than in the barotropic component and at the altitude of $10 \mathrm{~km}$ (see below). This difference is due to the different effects of the two mechanisms that spin up the jet, as discussed in section $2 b$.

Daily snapshots of the streamfunction evolution at an altitude of $10 \mathrm{~km}$ are shown in the bottow row of Fig. 12. The spatial patterns are very similar to those of the barotropic component but the jet and the eddies are much stronger in the western part of the domain. This effect is due to the baroclinic component that gives rise to the baroclinic instability shown in the second row, and to the formation of the eddies. The jet's speed at $10 \mathrm{~km}$ reaches values of $18 \mathrm{~ms}^{-1}$ and its length is up to $5000 \mathrm{~km}$ during the intervals of most vigorous flow. Easterlies appear in the model simulation south of $30 \mathrm{~N}$.

The limit to the length of zonal jets follows from the theory of geostrophic turbulence (Charney 1971) in the presence of the $\beta$-effect (Rhines 1975). Salmon (1998, Section 6.3) describes, in particular, the effect of localized mid-latitude stirring of an initially quiescent flow in a periodic $\beta$-channel. In our case, the localized stirring is provided by the finite-length, SWNE-oriented SST front. Considerations of energy and potential enstrophy conservation predict that the resulting flow is strongly eastward near the latitude of the stirring and weakly westward everywhere else; see Eq. (6.3.19) and Fig. 6.4 in Salmon (1998). FGS04 found that the jet length in their simulations and the theoretical formula agree quite well; see Table 2 there.

\section{b. Spectral analysis}

In order to focus on interannual oscillations, periods shorter than or equal to $1 \mathrm{yr}$ were again removed by applying a 12-month running average to the model simulations. MSSA was then 
applied to the filtered data with a window width of $M=150$ month. As for the SODA dataset, only those signals that are significant at the $95 \%$ level will be analyzed further below. We will analyze here the barotropic and baroclinic components, as well as the surface streamfunction.

We first applied MC-MSSA with a window of $M=150$ months to the barotropic and baroclinic components together; the resulting MC-MSSA spectrum is shown in Fig. 13. The first two ST-EOFs are associated with a nonlinear trend and have a variance of $23 \%$ (not shown). The leading oscillatory mode is captured by ST-EOFs $(3,4)$, it accounts for a variance of $15 \%$ and it has a nominal period of $8.2 \mathrm{yr}$. However, examination of the maximum entropy spectrum (Childers 1978; Penland et al. 1991; Ghil et al. 2002a) of the leading PCs obtained by a conventional EOF analysis of the same fields (not shown) reveals a broad peak in the 7.8-10 yr band. This broad peak brackets the 8.5-yr and 10.5-yr oscillations identified in the SST field in the CHR (Fig. 7) and GBR (Fig. 11) regions, respectively, and might be interpreted as an integrated atmospheric response to them. In section 5 below we show that this atmospheric peak disappears from the model circulation when we filter out the se two SST oscillations from the SODA dataset.

Phase composites of the RCs of the 7.8-10-year mode in the first half of the period are shown in Figs. 14-16a for the barotropic, baroclinic, and surface streamfunctions respectively; the counterparts of these phase composites at $10-\mathrm{km}$ altitude (not shown) resemble the barotropic ones in Fig. 14. The anomalies in the barotropic mode exhibit an intense jet during the phases labeled "time $=2.0 \mathrm{yr}$ " and "time $=3.1 \mathrm{yr}$ " over the CHR and GBR regions (compare Fig. 1), where the Gulf Stream is most variable intense, and they are substantial over the whole domain. South of $30 \mathrm{~N}$, the flow in the anomaly changes to a westward direction, and might explain the westward propagation of this mode around the globe as a broad jet whose 
intensity oscillates in time. (Feliks et al. 2010a). for instance, found oscillations with a period of 7-8 yr over the Eastern Mediterranean and even the Ethiopian Plateau.

In the baroclinic mode (Fig. 15), the largest amplitudes are present at the same time as in the barotropic mode. Moreover, a strong anticyclonic eddy is found in the western part of the domain, and a large, but weak cyclonic gyre covers the rest of the domain. This gyre has a weak eastward flow in its southern part, between latitudes $15 \mathrm{~N}$ and $25 \mathrm{~N}$, and westward flow in its northern part, between $35 \mathrm{~N}$ and $50 \mathrm{~N}$.

In the sensitivity experiment in which the width of the sponge layer was $\Delta L=750 \mathrm{~km}$, no significant differences between this solution and the one with $\Delta L=1000 \mathrm{~km}$ was found. In the second experiment, with $\Delta L=500 \mathrm{~km}$, the solution became unstable after 35 years. The analysis was carried out over these 35 years and the main features in the mean circulation and in the interannual oscillatory modes, like those appearing in Fig. 15 near the western boundary, look very similar to those for $\Delta L=1000 \mathrm{~km}$ or $\Delta L=750 \mathrm{~km}$, although the 8.5 -year mode had less than 4 full cycles. We thus conclude that the width $\Delta L$ of the sponge layer affects but little the main spatio-temporal features of the solution, but is quite important in maintaining its stability.

The seasonal-cycle mode near the surface is shown in Fig. 16b. In the summer, the seasonal-cycle anomaly reduces the mean flow, which is therefore weak on average. In the winter, the seasonal-cycle anomaly enhances the mean flow, which is therefore strong in this season. The amplitude of the seasonal mode is larger by an order of magnitude than the amplitude of the 7.5-10-yr mode and the snapshots shown in Fig. 12 are, therefore, mainly influenced by the seasonal cycle. While the interannual modes have a smaller direct influence on the large-scale atmospheric flow than the march of the seasons, their effect on weather 
variability from year to year can still be significant, as suggested by the documented impact of the NAO phase on weather phenomena near and far from the North Atlantic (Hurrell et al. 2003; Feliks et al. 2010a).

The other significant ST-EOFs, ordered by decreasing variance, along with the associated periods and variances are the following: ST-EOFs $(5,6)$ have a dominant period of $2.9 \mathrm{yr}$ and a variance of $12 \%$; ST-EOFs $(7,8)$ have a dominant period of $3.8 \mathrm{yr}$ and a variance of $9 \%$; ST-EOFs $(9,10)$ have a dominant period of $2.5 \mathrm{yr}$ and a variance of $7 \%$. The phase composites of these oscillatory modes have spatial patterns that resemble those of the 7.8-10year mode but with smaller amplitude. The similarity of the spatial patterns of the four statistically significant atmospheric oscillatory modes with different periods is consistent with the similar structure of the oscillatory modes in the SST fields.

\section{c. Comparison with the observed NAO index}

The central hypothesis of this paper is that the interannual oscillations found in observed atmospheric data over the North Atlantic are spun up by interannual oscillations of the Gulf Stream front. To further support the results reported above, we next compare the oscillations found in our atmospheric model simulations with an analysis of the observed NAO index.

The NAO has been studied extensively over the last two decades (Hurrell et al. 2003), and its strength has been quantified using several indices. We use here the monthly index over the years 1823-2007, as compiled by Jones et al. (1997); the later, updated version is given at http://uww.cru.uea.ac.uk/ timo/projpages/nao update.htm. This index is defined by the difference between the normalized sea level pressure at Ponta Delgada, Azores, Portugal (37.7 $\mathrm{N}, 25.7 \mathrm{~W})$ and the normalized sea level pressure over Iceland $(65.7 \mathrm{~N}, 18.1 \mathrm{~W})$. 
Applying SSA to this index, Feliks et al. (2010a) identified significant oscillatory modes with periods of 7.7, 5.5, 4.0, 3.2 and 2.2 yr. Rogers (1984), Hurrel and Van Loon (1997), Robertson (2001), Gámiz-Fortis et al. (2002), and Palu`s and Novotná (2004) also found an oscillation with a period of about $8 \mathrm{yr}$ in the NAO index. A quasi-biennial component is present in the closely related Arctic Oscillation, constructed from hemispheric sea level pressures, as well (Trenberth and Paolino 1981; Robertson 2001).

We compare here the observed NAO index to its counterpart in the atmospheric model simulations, defined as follows. First we compute the model's surface pressure, equivalent to the streamfunction at the surface level, $z=0$. The simulated NAO index (SNI) is then defined to be the difference in the model's surface pressure anomalies - i.e., the deviation from the 50-yr mean of the simulation — between the points $(41 \mathrm{~N}, 27 \mathrm{~W})$ and $(59 \mathrm{~N}, 29 \mathrm{~W})$; these two points are shown in Fig. 16 as points B and A. For these two model points, the correlation between the SNI and observed NAO index is 0.52 , i.e. very close to the maximum value obtained when defining the model's SNI as the difference between any two points in the domain; the longitude of point A defining the SNI is located about 10 degrees west of the northern point defining the NAO index. This difference is due to the geometric distortion by the $\beta$-plane used in the atmospheric model: longitude $29 \mathrm{~W}$ on the $\beta$-plane at latitude $59 \mathrm{~N}$ corresponds to a longitude of $19 \mathrm{~W}$ on the globe, i.e. point A does lie inside the model's Iceland.

In the following analysis, we again filtered out first the variability associated with periods shorter than or equal to $1 \mathrm{yr}$ by applying a 12-month running average to each time series (Fig. 17a). We then applied a bivariate MSSA to the NAO index (channel 1) and to the SNI index (channel 2), with a window width of $M=200$ months, normalizing each index by its respective variance. ST-EOF pair $(3,4)$ captures the lowest-frequency components, including 
the nonlinear trend (Ghil et al. 2002a), in both indices. To isolate any underlying low-frequency oscillations, we applied MSSA to the detrended indices with the same window width.

The MC-MSSA spectrum is shown in Fig. 18, and only those signals that are significant at the indicated (two-sided) 95\% level will be analyzed further below The RCs corresponding to the three significant ST-EOF pairs, ordered by decreasing variance, along with the associated periods and variances are shown in Fig. 17. These three modes of covariability between the observed and simulated indices capture $60 \%$ of the covariance and show high visual similarity between the observed NAO index and the SNI on these time scales.

We analyzed these three oscillatory modes further, in order to quantify their degree of synchronization, following Feliks et al. (2010a). For each oscillatory mode, this analysis measures the correlation of the amplitude modulation between the observed NAO index and the SNI, the degree of phase synchronization, and the ratio $\varepsilon$ of the energy that is contained in each of the two indices, $\varepsilon=E\left(R C_{\mathrm{SNI}}\right) / E\left(R C_{\mathrm{NAO}}\right)$; the energy $E$ of a pair of RCs is simply defined as the corresponding variance.

For the 8.5-yr oscillatory mode, there is complete synchronization of frequency, phase and amplitude between the observed NAO index and the SNI, i.e., in the terminology of Feliks et al. (2010a), the synchronization level is FPA: the correlation $r$ between their modulated amplitudes is of $r=0.99$; the difference $\sigma$ between their phases, $\sigma=0.11$ radian, is very small; and their energies are also very similar, with a ratio of $\varepsilon=1.08$. The synchronization of the 2.8 yr mode is also of type FPA, with $r=0.91, \sigma=0.19$ radian, and an energy ratio of $\varepsilon=1.15$. The 4.5-yr mode is marginally synchronized in frequency and phase - i.e., of FP type, following Feliks et al. (2010a) — with $r=0.87$ and $\sigma=0.72$, while the energy in SNI is significantly larger than in the observed NAO index, $\varepsilon=5.6$. 
The above synchronization analysis provides further quantitative evidence of the high degree of similarity between the interannual modes of the observed NAO index and the SNI, and by inference for the role of the Gulf Stream front in driving these oscillations in the observed NAO. The direct connection between the 8.5-yr oscillatory mode in the SST field of the SODA reanalysis and that of the observed NAO index is shown in Fig. 17b. In this panel, the 8.5-yr mode in SST is plotted (dotted line) at the point $(37 \mathrm{~N}, 74 \mathrm{~W})$, where this anomaly reaches its maximum amplitude; this mode is also shown in Fig. 6 (dashed line there). There's a particularly good fit for 1972-2002; the divergence after that is probably due to an edge effect, since in dimensional units $M=16.7 \mathrm{yr}$. The correlation between SNI and SST in their amplitude modulation is $r=0.79$.

\section{d. The atmospheric circulation for spatially and temporally smoothed SST fields}

In the previous sections we have made the case that the interannual oscillations in the observed NAO index can be attributed to similar oscillations found in the Gulf Stream front. It might be countered that this is merely an association, and that our atmospheric model is responding, in fact, to large-scale SST variability imprinted in the SODA dataset by the history of the atmospheric circulation's variability over the North Atlantic. We provide further evidence in this subsection that the Gulf Stream front does play a key role in driving the variability of the atmospheric circulation. To do so, we reran the MABL-QG model with $\nabla^{2} T=0$ in the spherical rectangle (32N-50N, $85 \mathrm{~W}-40 \mathrm{~W})$, which includes both the CHR and GBR regions of the front (see again the boxes in Fig. 1). In this simulation, therefore, the Gulf Stream front cannot impact the model atmosphere, whose forcing derives only from the surrounding regions. 
The atmospheric circulation in this experiment changes drastically: both the jet and its associate eddies disappear completely. Repeating the MSSA analysis of section $4 \mathrm{~b}$, there were no statistically significant oscillations in the $7.8-10-\mathrm{yr}$ range; there are some statistically significant higher-frequency oscillations (Fig. 19), but none has a period longer than 3 yr and their energy is smaller by an order of magnitude than in the control experiment (Fig. 13).

In the next experiment we spatially smoothed the oceanic SST front to be similar to those used by typical GCMs, by filtering out wavelengths shorter than $400 \mathrm{~km}$. We forced the atmospheric model with the smoothed SST field. Snapshots of the atmospheric circulation so obtained are shown in Fig. 20; they are much weaker and miss entirely the strong jet and eddies when compared to the control experiment, as shown in Fig. 12. In fact, unlike in Fig. 12, there is barely a difference between the weakest jet (time $=1993.8)$ and the strongest o ne (time = 1996.3). The MSSA analysis was not able to identify statistically significant oscillations in the 7.8-10-yr range, although here, too, there are some statistically significant higher-frequency oscillations (Fig. 21) whose energy is smaller by an order of magnitude than in Fig. 13.

In yet another experiment, we forced the model atmosphere over the entire domain of Fig. 1, but with the SST variability low-pass-filtered in time so that no periods longer than 2 yr wrere present; note that the time-mean SST was included in the forcing. Snapshots of the atmospheric circulation in this integration (not shown) were found to be very similar to those obtained in the control run, with the mean fields also being very similar to those shown in Figs. 14-16 (last panels). In contrast to the control experiment, though, the MSSA analysis was not able to identify statistically significant oscillations in the 7.8-10-yr range (Fig. 22). As in Figs. 5,13 and 21 , there is a statistically significant peak at $3.8 \mathrm{yr}$, as well as some higher-frequency 
oscillations, but their energy is again smaller by an order of magnitude than in the control experiment (Fig. 13).

From these three experiments we conclude that, when the SST field is either spatially smoothed or the 7.8-10-yr peak is removed from it, then the $7.8-10-y r$ oscillations are also eliminated from the atmospheris circulation. It follows that we can indeed attribute the 7.8-10yr oscillations in the atmosphere to similar oscillations in the SST front.

\section{Concluding remarks}

\section{a. Summary}

The cause of interannual oscillations in the extratropical atmosphere is a matter of longstanding arguments. There is substantial consensus on the extratropical effects of ENSO, but these do not extend to periods longer than 4-5 yr. Several theories exist for the well-known 78-yr peak in the NAO (e.g., Deser and Blackmon 1993; Dettinger et al. 1995; Plaut et al. 1995; Moron et al. 1998; Wunsch 1999; Da Costa et al. 2002; Hurrell et al. 2003; Feliks et al. 2010a), and they were briefly summarized in the Introduction. In this paper, we showed that sharp SST fronts associated with the Gulf Stream can spin up an atmospheric jet over the North Atlantic, and give rise to oscillations with such a period in a simplified atmospheric model.

First, we analyzed in section 3 monthly SST and SSH fields from the 50 years (19582007) of the SODA reanalysis dataset, over the North Atlantic region shown in Fig. 1. We found two regions along the Gulf Stream front that have prominent and statistically significant narrow-band interannual oscillations, namely the region just downstream of Cape Hatteras (CHR), and in the Grand Banks region (GBR), centered at (44 N, $47 \mathrm{~W}$ ). Several statistically 
significant oscillatory modes were found in these two regions; their periods include an 8.5-yr mode in the CHR region, and a 10.5-yr mode in the GBR region (Figs. 5 and 9, respectively).

The spatio-temporal pattern of the 8.5-yr mode in both the SST and SSH fields within the CHR region (Figs. 7 and 8, respectively) shares certain features with the so-called gyre mode that characterizes the interannual variability in the double-gyre problem of the midlatitude wind-driven ocean circulation (Jiang et al. 1995; Dijkstra and Ghil 2005). This gyre mode was found to have a dominant 7-8-yr peak across a hierarchy of ocean models, QG and shallow-water, barotropic and baroclinic (Speich et al., 1995; Ghil et al., 2002b; Simonnet and Dijkstra 2002; Simonnet et al., 2003a, 2003b, 2005; Dijkstra and Ghil, 2005).

Shorter-period SST oscillations were also identified with periods of 3.7, 4.5, and $6.2 \mathrm{yr}$ in the CHR region, and with 3.2- and 5.7-yr periods in the GBR region. The spatial patterns of all these oscillatory modes include a large-scale Gulf Steam meander off Cape Hatteras, as well as extension-and-contraction of the jet-like feature in the GBR region. These two basic spatiotemporal patterns characterize all the significant oscillations identified in the respective regions.

This similarity might seem surprising, given the fact that - in the free oscillations of a vibrating string or drumhead — higher-frequency oscillations are typically associated with smaller spatial scales. The difference is due to the fact that these oscillations in the western North Atlantic are strongly constrained by the bathymetry, on the one hand, and the prevailing wind pattern, on the other. The latter, of course, is affected in turn by the oceanic variability, as we have seen in section 4.

Following upon the results of Feliks et al. (2004, 2007; abbreviated as FGS04 and FGS07), we used in section 4 the SST monthly history from the SODA dataset to drive the atmospheric model of FGS07 through the Laplacian of the SST field, cf. Eq. (3). In the 
resulting atmospheric simulations, in which $\nabla^{2} T$ was the sole model forcing, we identified two extreme states, consisting of (i) an extended, strong jet state, and (ii) a state of very weak atmospheric flow with no jet, respectively (Fig. 12). In the former, a strong jet develops over the oceanic SST front, in which the jet extends further eastward and reaches a length of 5000 $\mathrm{km}$ and a speed of $18 \mathrm{~ms}^{-1}$. This jet is most prominent in the model's upper layer.

Prominent interannual oscillatory modes were found in the model atmosphere, with a spatial pattern characteristic of the strong-jet state, with anticyclonic anomalies to the south of the jet and cyclonic ones to the north; both these features extend throughout the model's North Atlantic domain. The statistically significant oscillatory modes in the model's free atmosphere have periods of 7.8-10 yr, $3.8 \mathrm{yr}$ and $2.9 \mathrm{yr}$ (Fig. 13). This range of periods corresponds approximately to that of the oscillatory modes found in the SST front of the CHR and GBR regions, although the periods do not correspond exactly. The $7.8-10-\mathrm{yr}$ mode in the model atmosphere is broad compared to the 8.5-yr and 10.5-yr modes identified in the SST front of the CHR and GBR regions, respectively, and may arise as a result of the nonlinear interaction between the two. Our results suggest that the spin- up of the 3.8-yr mode may be induced by more localized SST oscillation in the CHR region.

\section{b. Discussion}

A striking results of this study is the close similarity between the observed NAO index and the model's simulated NAO index (SNI), cf. section 4c (Fig. 17). Comparing the observed NAO index with the SNI, we found that the overall correlation is $r=0.52$. The complete synchronization of the 8.5-yr oscillatory mode in the observed and simulated indices leads us to the conclusion that this NAO mode is induced by the mode with the same or very similar periodicity in the Gulf Stream front. This result is at least consistent with the review of Small et 
al. (2008), in which they emphasize that in frontal zones and over eddies, it is indeed the ocean that drives the atmosphere, rather than the other way around; see also Manganello (2008).

The spatio-temporal pattern of the 8.5-yr mode in SSTs and SSHs shares certain features with the so-called gyre mode that characterizes the interannual variability in the double-gyre problem of the mid-latitude wind-driven ocean circulation (Jiang et al. 1995; Simonnet and Dijkstra 2002; Simonnet et al. 2003a, b, 2005). This gyre mode was found to have a dominant 7-8-yr peak across a hierarchy of ocean models, QG and shallow-water, barotropic and baroclinic (Speich et al. 1995; Chang et al. 2001; Ghil et al. 2002b; Dijkstra and Ghil (2005).

The gyre mode was shown to be robust with respect to forcing by the seasonal cycle in wind stress (Sushama et al. 2007) and had been found in a century of SST data for the North Atlantic (Moron et al. 1998). Several other authors, going back to Veronis $(1963,1966)$, found intrinsic multimodality or interannual variability in the oceans' wind-driven circulation, even when the wind-stress forcing is constant in time (Cessi and Ierley, 1995; Meacham 2000; Nadiga and Luce 2001; Nauw and Dijkstra 2001); more complete references can be found in Dijkstra (2005) and Dijkstra and Ghil 2005). It would seem that the results of the pre sent paper provide substantial evidence in support of such intrinsic ocean variability, in general, and the gyre mode, in particular, giving rise to near-decadal variability in the Gulf Stream, on the one hand, and in the NAO, on the other.

Further evidence that the 7-8-yr atmospheric mode is indeed induced by the neardecadal oscillations of the Gulf Stream front in the CHR and GBR regions is provided by three additional experiments. In these atmospheric-model experiments, the SST front was (a) removed entirely; (b) spatially smoothed; or (c) low-pass-filtered in time to remove the 
oscillations with periods longer than $3 \mathrm{yr}$. In all three cases, the 7-8-yr atmospheric mode disappeared, and other interannual oscillations were greatly attenuated.

If these findings are further confirmed by high-resolution GCM computations and observations - in the same way in which Minobe et al. (2008) confirmed those of FGS04 and FGS07 — one could expect to find some predictive skill in the periodicity of the oceanic mode first, and the atmospheric one thereafter. In FGS04 it was clearly shown that the imposed 7-yr periodicity in the SST front's strength resulted in substantial modulation of intraseasonal activity in the atmosphere above. Thus one might be able to anticipate the frequency and severity of extreme events over Europe (e.g., Yiou and Nogaj 2004) from a monitoring of the Gulf Stream.

Acknowledgments. It is a pleasure to acknowledge many useful discussions with Eric Simonnet and the constructive comments of two anonymous referees. This work was supported by U.S. Department of Energy grants DE-FG02-02ER63413 and DE-FG02-07ER64439 at UCLA and DE-FG02-07ER64429 at the IRI. 


\section{References}

Allen, M. R., and L. A. Smith, 1996: Monte Carlo SSA: Detecting irregular oscillations in the presence of coloured noise, J. Clim., 9, 3373-3404.

Bjerknes, J., 1964: Atlantic air-sea interaction. Advances in Geophysics, Vol. 10, Academic Press, 1-82.

Broomhead, D.S., and G.P. King, 1986a: Extracting qualitative dynamics from experimental data. Physica D, 20, 217-236.

Broomhead, D. S., and G. P. King, 1986b: On the qualitative analysis of experimental dynamical systems. In Nonlinear Phenomena and Chaos, Sarkar S (Ed.), Adam Hilger, Bristol, pp. 113-144.

Carton, J. A. and B. S. Giese, 2008: A reanalysis of ocean climate using Simple Ocean Data Assimilation (SODA). Mon. Wea. Rev., 136, 2999-3017.

Chang, K.-I., M. Ghil, K. Ide, and C.-C. A. Lai, 2001: Transition to aperiodic variability in a wind-driven double-gyre circulation model, J. Phys. Oceanogr., 31, 1260-1286.

Chao, Y., M. Ghil, and J. C. McWilliams, 2000: Pacific interdecadal variability in this century's sea surface temperatures, Geophys. Res. Lett., 27, 2261-2264.

Charney, J. G., 1971: Geostrophic turbulence. J. Atmos. Sci., 28, 1087-1095.

Childers, D. G. (Ed.), 1978: Modern Spectrum Analysis, IEEE Press, Piscataway, N. J., 331 pp.

Da Costa, E. D., and A. Colin de Verdière, 2002: The 7.7-year North Atlantic oscillation, Q. J. R. Meteorol. Soc., 128A, 797-817.

Deser, C., and M. L. Blackman, 1993: Surface climate variation over the North Atlantic Ocean during winter: 1900-1999, J. Clim., 6, 1743-1753.

Dettinger, M. D., M. Ghil and C. L. Keppenne, 1995: Interannual and interdecadal variability in United States surface-air temperatures, 1910-87, Climatic Change, 31, 35-66.

Dijkstra, H. A. 2005. Nonlinear Physical Oceanography: A Dynamical Systems Approach to the Large Scale Ocean Circulation and El Niño. 2nd ed., Kluwer Academic Publishers, Dordrecht, the Netherlands.

Dijkstra, H.A., and M. Ghil, 2005 : Low-frequency variability of the large-scale ocean circulation: A dynamical systems approach, Rev. Geophys., RG3002, doi:10.1029/2002RG000122. 
Feliks, Y., 1990: Isolated vortex evolution in 2 and 4 mode models. Deep-Sea Res., 37, 571591.

Feliks, Y., and M. Ghil, 1996: Mixed barotropic-baroclinic eddies growing on an eastward midlatitude jet. Geophys. Astrophys. Fluid Dyn., 82, 137-171.

Feliks, Y., M. Ghil and E. Simonnet, 2004: Low-frequency variability in the midlatitude atmosphere ind uced by an oceanic thermal front. J. Atmos. Sci., 61, 961-981.

Feliks, Y., M. Ghil, and E. Simonnet, 2007: Low-frequency variability in the mid-latitude baroclinic atmosphere induced by an oceanic thermal front, J. Atmos. Sci., 64, 97-116.

Feliks, Y., M. Ghil, and A. W. Robertson, 2010a: Oscillatory climate modes in the Eastern Mediterranean and their synchronization with the NAO, J. Clim., accepted.

Feliks, Y., E. Tziperman and B. Farrell, 2010b: Non normal frontal dynamics, J. Atmos. Sci., 67, 1218-1231.

Flierl, G. R., 1978: Models of vertical structure and the calibration of two-layer models. Dyn. Atmos. Oceans, 2, 341-381.

Ghil, M., M. R. Allen, M. D. Dettinger, K. Ide, D. Kondrashov, M. E. Mann, A. W. Robertson, A. Saunders, Y. Tian, F. Varadi, and P. Yiou, 2002a: Advanced spectral methods for climatic time series, Rev. Geophys., 40(1), pp. 3.1-3.41, doi: 10.1029/2000RG000092.

Ghil, M., Y. Feliks, and L. Sushama, 2002b: Baroclinic and barotropic aspects of the winddriven ocean circulation, Physica $D, 167,1-35$.

Haidvogel, D. B., A. R. Robinson, and E. E. Schulman, 1980: The accuracy, efficiency and stability of three numerical models with application to open ocean problems. J. Comput. Phys., 34, 1-53.

Holton, J. R., 1992: An Introduction to Dynamic Meteorology, 3d ed., Academic Press, 511 pp.

Hurrell, J.W., 1995: Decadal trends in the North Atlantic Oscillation: Regional temperatures and precipitation, Science, 676-679.

Hurrell, J.W., and H. V. Loon, 1997: Decadal variations in climate associated with the North Atlantic oscillation. Climatic Change 36: 301-326.

Hurrell, J. W., Y. Kushnir, G. Ottersen, and M. Visbeck, 2003: An overview of the North Atlantic Oscillation. The North Atlantic Oscillation: Climatic Significance and Environmental Impact, Geophys. Monogr., Vol. 134, American Geophysical Union, pp. 1-35. 
Jiang, S., F.-F. Jin, and M. Ghil, 1995: Multiple equilibria, periodic, and aperiodic solutions in a wind-driven, double-gyre, shallow-water model, J. Phys. Oceanogr., 25, 764-786.

Jones, P.D., T. Jonsson, and D. Wheeler, 1997: Extension to the North Atlantic Oscillation using early instrumental pressure observations from Gibraltar and South-West Iceland. Int. J. Climatol. 17, 1433-1450.

Joyce, M. T., C. Deser and M. A. Spall, 2000: The relation between decadal variability of subtropical mode water and the North Atlantic Oscillation. J. Climate, 13, 2550-2569.

Keppenne, C. L., and M. Ghil, 1993: Adaptive filtering and prediction of noisy multivariate signals: An application to subannual variability in atmospheric angular momentum, Intl. J. Bifurcation \& Chaos, 3, 625-634.

Kravtsov, S., A. W. Robertson, and M. Ghil, 2005: Bimodal behavior in the zonal mean flow of a baroclinic-channel model. J. Atmos. Sci., 62, 1746-1769.

Kushnir, Y., W. A. Robinson, I. Bladé, N. M. J. Hall, S. Peng, and R. Sutton, 2002: Atmospheric GCM response to extratropical SST anomalies: Synthesis and evolution. $J$. Climate, 15, 2233-2256.

Manganello, J. V., 2008: The influence of sea surface temperature anomalies on low-frequency variability of the North Atlantic Oscillation, Clim. Dyn., 30, 621-641.

Meacham, S. P. (2000), Low-frequency variability of the wind-driven circulation, J. Phys. Oceanogr., 30, 269-293.

Minobe, S., A. Kuwano-Yoshida, N. Komori, S. P. Xie, and R. J. Small, 2008: Influence of the Gulf Stream on the troposphere. Nature, 452, 206-209.

Moron, V., R. Vautard, and M. Ghil, 1998: Trends, interdecadal and interannual oscillations in global sea-surface temperatures, Clim. Dyn., 14, 545-569.

Nadiga, B. T., and B. Luce (2001), Global bifurcation of Shilnikov type in a double-gyre model, J. Phys. Oceanogr., 31, 2669-2690.

Nauw, J., and H. A. Dijkstra, 2001: The origin of low-frequency variability of double-gyre wind-driven flows, J. Mar. Res., 59, 567-597.

Özgökmen, T., E. P. Chassignet and A. M. Priva, 1997: Impact of wind forcing, bottom topography, and inertia on midlatitude jet separation in a quasigeostrophic model. J. Phys. Oceanogr., 27, 2460-2476.

Pedlosky, J., 1987: Geophysical Fluid Dynamics, 2nd ed. Springer-Verlag, 710 pp. 
Penland, C., M. Ghil, and K. M. Weickmann, 1991: Adaptive filtering and maximum entropy spectra, with application to changes in atmospheric angular momentum, J. Geophys. Res., 96, 22659-22671.

Plaut, G., and R. Vautard, 1994: Spells of low-frequency oscillations and weather regimes in the Northern Hemisphere. J. Atmos. Sci., 51, 210-236.

Rhines, P. B., 1975: Waves and turbulence on a beta-plane. J. Fluid Mech., 69, 417-443.

Robertson, A. W., 2001: On the influence of ocean-atmosphere interaction on the Arctic Oscillation in two general circulation models. J. Climate, 14, 3240-3254.

Robertson, A. W., M. Ghil, and M. Latif, 2000: Interdecadal changes in atmospheric lowfrequency variability with and without boundary forcing. J. Atmos. Sci., 57, 1132-1140.

Rossby, T., 1999: On gyre interactions: Part II. Deep-Sea Research, 46, 139-164.

Salmon, R., 1998: Lectures on Geophysical Fluid Dynamics. Oxford University Press, 378 pp.

Simonnet, E., and H. A. Dijkstra, 2002: Spontaneous generation of low-frequency modes of variability in the wind-driven ocean circulation, J. Phys. Oceanogr., 32, 1747-1762.

Simonnet, E., M. Ghil, and H. A. Dijkstra, 2005: Homoclinic bifurcations in the quasigeostrophic double-gyre circulation, J. Mar. Res., 63, 931-956.

Simonnet, E., M. Ghil, K. Ide, R. Temam, and S. Wang, 2003a: Low-frequency variability in shallow-water models of the wind-driven ocean circulation. Part I: Steady-state solutions. $J$. Phys. Oceanogr., 33, 712-728.

Simonnet, E., M. Ghil, K. Ide, R. Temam, and S. Wang, 2003b: Low-frequency variability in shallow-water models of the wind-driven ocean circulation. Part II: Time-dependent solutions. J. Phys. Oceanogr., 33, 729-752.

Small, R.J., S.P. de Szoeke, S.P. Xie, L. O’Neill, H. Seo, Q. Song, P. Cornillon, M. Spall, and S. Minobe, 2008: Air-sea interaction over ocean fronts and eddies, Dyn. Atmos. Oceans, 45, 274 319.

Smith, R. D., J. K. Dukowicz, and R. C. Malone, 1992: Parallel ocean general circulation modeling. Physica D, 60, 38-61.

Speich, S., H. Dijkstra, and M. Ghil, 1995: Successive bifurcations in a shallow-water model, applied to the wind-driven ocean circulation. Nonlin. Proc. Geophys., 2, 241-268.

Sushama, L., M. Ghil, and K. Ide, 2007: Spatio-temporal variability in a mid-latitude ocean basin subject to periodic wind forcing. Atmosphere-Ocean, 45, 227-250, doi: $\underline{10.3137 / \mathrm{ao} .450404 .}$ 
Vautard, R., and M. Ghil, 1989: Singular spectrum analysis in nonlinear dynamics, with applications to paleoclimatic time series, Physica D, 35, 395-424.

Vautard, R., P. Yiou, and M. Ghil, 1992: Singular-spectrum analysis: A toolkit for short, noisy chaotic signals, Physica D, 58, 95-126.

Veronis, G., 1963: An analysis of wind-driven ocean circulation with a limited number of Fourier components. J. Atmos. Sci, 20, 577-593.

Veronis, G., 1966: Wind-driven ocean circulation. Part II: Numerical solution of the nonlinear problem. Deep-Sea Res., 13, 30-55.

Wunsch, C., 1999: The interpretation of short climate records, with comments on the North Atlantic and Southern Oscillations, Bull. Amer. Meteorol. Soc., 80, 245-255.

Yiou, P., and M. Nogaj, 2004: Extreme climatic events and weather regimes over the North Atlantic: When and where?, Geophys. Res. Lett., 31, L07 202, doi:10.1029/2003GL019119. 
TABLE 1. Characteristic scales and reference values for the atmospheric model.

\begin{tabular}{|l|c|}
\hline$L(\mathrm{~km})$ & 50 \\
\hline$H(\mathrm{~km})$ & 10 \\
\hline$H_{a(\mathrm{~km})}$ & 10 \\
\hline$H_{E}(\mathrm{~km})$ & 0.8 \\
\hline$T_{\bar{i}}(\mathrm{day})$ & 0.58 \\
\hline$V\left(\mathrm{~ms}^{-1}\right)$ & 1 \\
\hline$\beta\left(\mathrm{m}^{-1} \mathrm{~s}^{-1}\right)$ & $1.8 \times 10^{-11}$ \\
\hline$k_{0}\left(\mathrm{~m}^{2} \mathrm{~s}^{-1}\right)$ & 3.3 \\
\hline$K_{\mathrm{H}}\left(\mathrm{m}^{2} \mathrm{~s}^{-1}\right)$ & $10^{2}$ \\
\hline$f\left(\mathrm{~s}^{-1}\right)$ & $10^{-4}$ \\
\hline$g\left(\mathrm{~ms}^{-2}\right)$ & 9.81 \\
\hline$\theta_{0}(\mathrm{~K})$ & 300 \\
\hline$\rho_{0}\left(\mathrm{gcm}^{-3}\right)$ & $10^{-3}$ \\
\hline$L / \lambda_{1}(\mathrm{~km})$ & 575 \\
\hline
\end{tabular}




\section{List of Figures}

Fig. 1: Mean sea surface temperature (SST) field in the North Atlantic over the curvilinear rectangle $(15 \mathrm{~N}-65 \mathrm{~N}, 85 \mathrm{~W}-0 \mathrm{~W})$ from Simple Ocean Data Assimilation (SODA) data over the 50-yr interval from January 1958 to December 2007. The heavy solid lines are the land boundaries; contour interval $(\mathrm{CI})$ is $2.8 \mathrm{~K}$, and extreme values (max and $\min$ ) are given in the legend. Small rectangles indicate the two regions on which we concentrate in particular: (1) the Cape Hatteras region (CHR; 34 N-43.50 N, 75 W-60 W), and (2) the Grand Banks region (GBR; $42 \mathrm{~N}-50 \mathrm{~N}, 55 \mathrm{~W}-35 \mathrm{~W})$.

Fig. 2: Mean sea surface height (SSH) in the North Atlantic over the same area as in Fig. 1, from the monthly mean SODA data. Solid lines are positive and dashed lines are negative differences from mean sea level; $\mathrm{CI}=0.168 \mathrm{~m}$.

Fig. 3: (a) The mechanism for atmospheric jet generation proposed by Feliks et al. (2004; FGS04 for short): the oceanic jet (heavy blue arrow) gives rise to an atmospheric jet stream (black arrow above), accompanied by a double-gyre circulation (red and blue circles), up to the tropopause level; warm color indicates warm side of the oceanic front. (b) Snapshots of the thermal component $\alpha \nabla^{2} T$ (upper panels) of the vertical velocity $w\left(H_{E}\right)$, and of the mechanical component $\gamma \nabla^{2} \psi$ (lower panels), in the North Atlantic over the area of Fig. 1; red contours indicate upward velocity, i.e., anticyclonic rotation in the horizontal.

Fig. 4: Seasonal cycle of the SST field in the western North Atlantic, (32 N-48 N, 75 W-40 W), for the 50 years 1958-2007, with the year divided in eighths, rather than months. Composites of the 1-yr mode, in four phase categories, correspond to one half of the annual cycle, namely June-to-November; the phases are labeled by the mid-point of the phase category. The panels look very similar for the other half of the cycle, December-May, only 
with opposite sign; that is the spatial pattern in the panel for $0.625 \mathrm{yr}$ (not shown) $\approx-$ (panel for $0.125 \mathrm{yr})$, etc. The se composites are based on the M-SSA pair $(1,2)$, which captures $90 \%$ of the total variance (positive contours solid, negative ones dashed); $\mathrm{CI}=1 \mathrm{~K}$ for the se fo ur panels, and $\mathrm{CI}=1.7 \mathrm{~K}$ for the mean SST field (last panel).

Fig. 5: Spectral analysis of the SST evolution in the CHR region $(34 \mathrm{~N}-43.50 \mathrm{~N}, 75 \mathrm{~W}-$ $60 \mathrm{~W}$ ), using Monte Carlo MSSA (MC-MSSA) with a window width of $M=150$ months, after having subtracted the seasonal cycle. The estimated variance of each mode in the dataset is shown as a filled black square, while lower and upper ticks on the error bars indicate the 5th and 95 th percentiles of a red-noise process constructed from a surrogate data ensemble of 100 time series; each of the surrogates has the same variance and lag-one autocorrelation as the original record. The surrogate time series (Allen and Smith, 1996) were produced by projecting the first 10 principal components of the MSSA analysis onto the basis vectors of a red-noise process. Highly significant pairs are labeled by their period.

Fig. 6: The evolution of the reconstructed 8.5-yr oscillatory mode in SST, at the point (37 N, $74 \mathrm{~W}$ ), where its amplitude in the CHR region is maximal; raw data shown as the solid line, and RCs $(3,4)$, which capture the oscillation, as the dashed line.

Fig. 7: Composites of the 8.5-yr mode of SST in the CHR region, in four phase categories, which correspond to the first half of the period. These composites are based on the MSSA pair $(3,4)$, which captures $13 \%$ of the total variance (positive contours solid, negative ones dashed). The phase categories are labeled by their mid-point, as in Fig. 4, and CI $=0.15$ $\mathrm{K}$; the mean SST (last panel) has $\mathrm{CI}=1.7 \mathrm{~K}$. 
Fig. 8: Composites of the 8.5-yr mode of SSH in the CHR region; same ordering and labeling of panels, and same convention for contours, as in Fig. 7. The four phase composites are based on the M-SSA pair $(3,4)$, which captures $12 \%$ of the total variance; $\mathrm{CI}=0.02 \mathrm{~m}$ here, while $\mathrm{CI}=0.13 \mathrm{~m}$ for the mean SSH field (last panel).

Fig. 9: Spectral analys is of the SST evolution in the GBR region (42 N-50 N, 55 W-35 W), after detrending and by using MC-MSSA with a window width of $M=150$ months. Same conventions as in Fig. 5; surrogate time series produced the same way as there.

Fig. 10: The evolution of the reconstructed 10.5-yr oscillatory mode in SST, at the point (47 W, $44 \mathrm{~N}$ ), where its amplitude is maximal in the GBR region; raw data shown as the solid line, and RCs (1,2), which capture the oscillation, as the dashed line.

Fig. 11: Composites of the 10.5-yr mode of SST in the GBR region; same ordering and labeling of panels, and same convention for contours, as in Fig. 7. The four phase composites are based on the MSSA pair $(1,2)$, which captures $10 \%$ of the total variance; $\mathrm{CI}=0.15 \mathrm{~K}$ here, while $\mathrm{CI}=1.7 \mathrm{~K}$ for the mean SST field (last panel).

Fig. 12: Evolution of the atmospheric jet induced by the SODA history of the SST field in the coupled MABL-QG model of Feliks et al. (2007; FGS07 for short). Snapshots shown at unequally spaced times, with the time, as well as the maximum and minimum values given in the legend of each plot; positive contours solid, negative contours dashed. First row: barotropic component, $\mathrm{CI}=8$; second row: baroclinic component, $\mathrm{CI}=6$; third row: surface streamfunction, $\mathrm{CI}=10$; bottom row: streamfunction at an altitude of $10 \mathrm{~km}, \mathrm{CI}=10$; nondimensional units. 
Fig. 13: Spectral analysis of the atmospheric model's combined barotropic and baroclinic modes using MSSA. The MC-MSSA spectrum shown here is computed with a window width of $M=150$ months; other details as in Figs. 5 and 9.

Fig. 14: Composites of the 7.8-10-yr mode of the atmospheric model's barotropic component, in four phase categories, like for the oceanic composites in Figs. 4, 7, 8 and 11. These composites are based on the M-SSA pair $(3,4)$, which captures $15 \%$ of the total variance; here $\mathrm{CI}=0.25$. Note that this broad peak in the atmospheric model encompasses the narrower oceanic peaks at $8.5 \mathrm{yr}$ and $10.5 \mathrm{yr}$ in the CHR and GBR regions, respectively; see Figs. 5 and 9. The mean field (last panel) has $\mathrm{CI}=4.9$ (nondimensional units).

Fig. 15: Same as Fig. 14 but for the baroclinic component; $\mathrm{CI}=0.15$ for the four composites and $\mathrm{CI}=2.3$ for the mean.

Fig. 16: Streamfunction field near the surface. (a) Composites of the $7.8-10-y r$ mode, as for Figs. 14 and 15; $\mathrm{CI}=0.35$ for the four composites and $\mathrm{CI}=6.0$ for the mean. For greater legibility, the average value of the field over the entire integration domain $(\psi=21)$ has been subtracted from the mean-field panel; this yields an approximately equal number of dashed and solid contours, rather than a preponderance of dashed ones. (b) Composites of the seasonal cycle, i.e. the 1-yr mode, of the atmospheric model, in four phase categories; same phases and conventions as in Fig. 4, for the oceanic composites. These composites are based on the M-SSA pair $(1,2)$, which captures $40 \%$ of the total variance; here $\mathrm{CI}=4$.

Fig. 17: The observed NAO index (dashed line) and simulated NAO index (SNI; solid line): (a) 12-month running mean; and (b-d) the RCs of the significant oscillatory modes in both the observed and simulated NAO indices, ordered by decreasing variance, along with the associated periods and variances. The dotted line in panel (b) is the reconstructed 8.5-yr 
oscillatory mode in the SST at the point $(37 \mathrm{~N}, 74 \mathrm{~W})$ that was shown already in Fig. 6; it is divided here by 45.6, for proper scaling to the nondimensional RCs.

Fig. 18: Spectral analysis of the observed NAO index and the atmospheric model's SNI, obtained by MC-MSSA with a window width of $M=200$ months; other details as in Fig. 9.

Fig. 19: Same as Fig. 13, but when the atmospheric model is driven by a flat SST field, with $\nabla^{2} T=0$, in the domain $(32 \mathrm{~N}-50 \mathrm{~N}, 85 \mathrm{~W}-40 \mathrm{~W})$; MC-MSSA spectrum computed with a window width of $M=150$ months, and same conventions as in Figs. 9 and 13 .

Fig. 20: Evolution of the atmospheric streamfunction at an altitude of $10 \mathrm{~km}$, but when the atmospheric model is driven by a spatially smoothed SST field; see text for details. The two snapshots are shown at the times of weakest and strongest jet, respectively; same CI and other conventions as in the lowermost row of Fig. 12.

Fig. 21: Spectral analysis of the atmospheric model's combined barotropic and baroclinic modes but when the atmospheric model is driven by a spatially smoothed SST field; see text for details. MC-MSSA spectrum computed with a window width of $M=150$ months, and same conventions as in Figs. 9 and 13.

Fig. 22: Spectral analysis of the atmospheric model's combined barotropic and baroclinic modes, as induced by the SST history from SODA but filtering out periods longer than 2 years. MC-MSSA spectrum computed with a window width of $M=150$ months, and same conventions as in Figs. 9 and 13. 


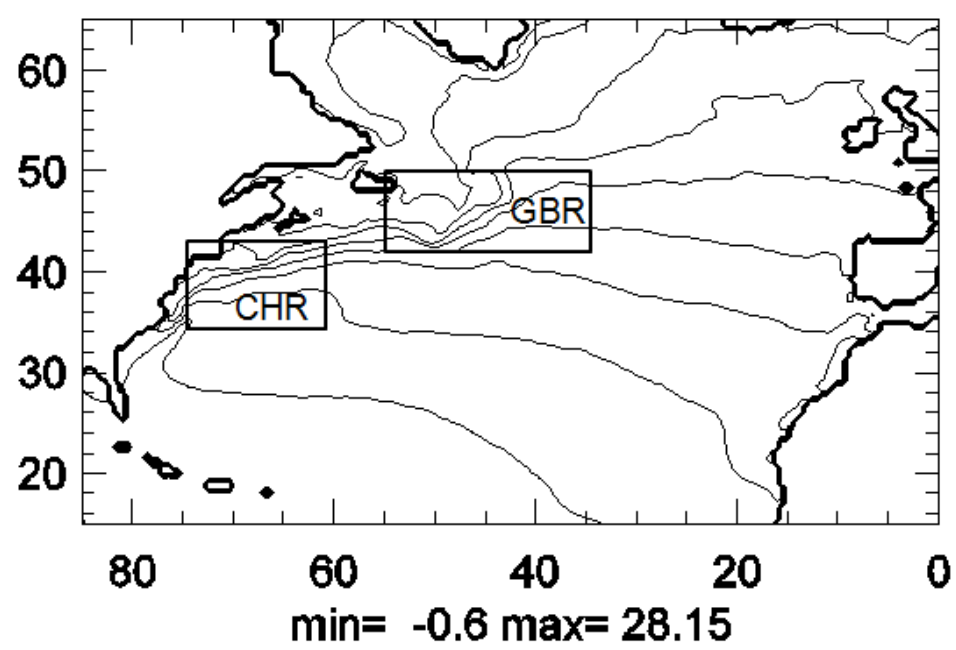

Fig. 1: Mean sea surface temperature (SST) field in the North Atlantic over the curvilinear rectangle (15 N-65 N, $85 \mathrm{~W}-0 \mathrm{~W})$ from Simple Ocean Data Assimilation (SODA) data over the 50-yr interval from January 1958 to December 2007. The heavy solid lines are the land boundaries; contour interval $(\mathrm{CI})$ is $2.8 \mathrm{~K}$, and extreme values (max and $\min$ ) are given in the legend. Small rectangles indicate the two regions on which we concentrate in particular: (1) the Cape Hatteras region (CHR; $34 \mathrm{~N}-43.50 \mathrm{~N}, 75 \mathrm{~W}-60 \mathrm{~W}$ ), and (2) the Grand Banks region (GBR; $42 \mathrm{~N}-50 \mathrm{~N}, 55 \mathrm{~W}-35 \mathrm{~W}$ ). 


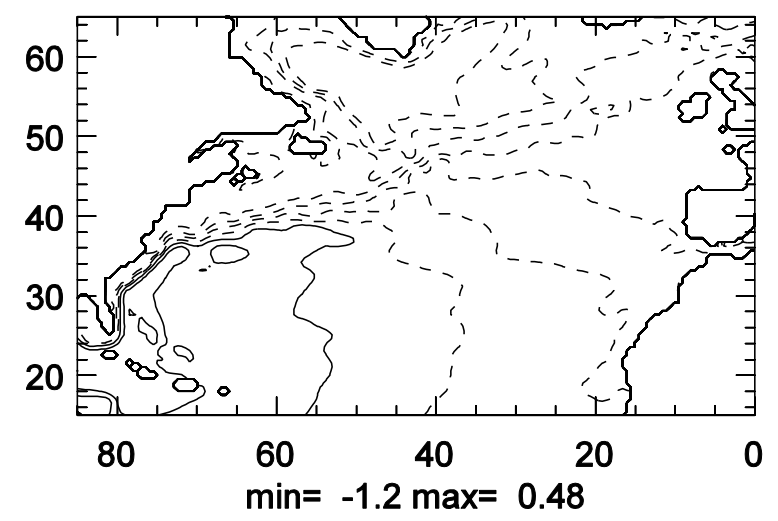

Fig. 2: Mean sea surface height (SSH) in the North Atlantic over the same area as in Fig. 1, from the monthly mean SODA data. Solid lines are positive and dashed lines are negative differences from mean sea level; $\mathrm{CI}=0.168 \mathrm{~m}$. 
(a)

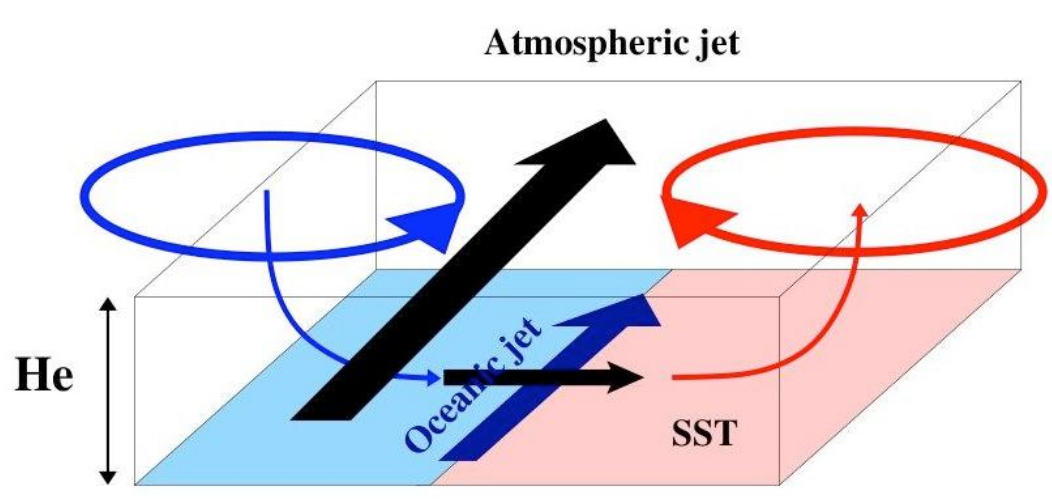

(b)
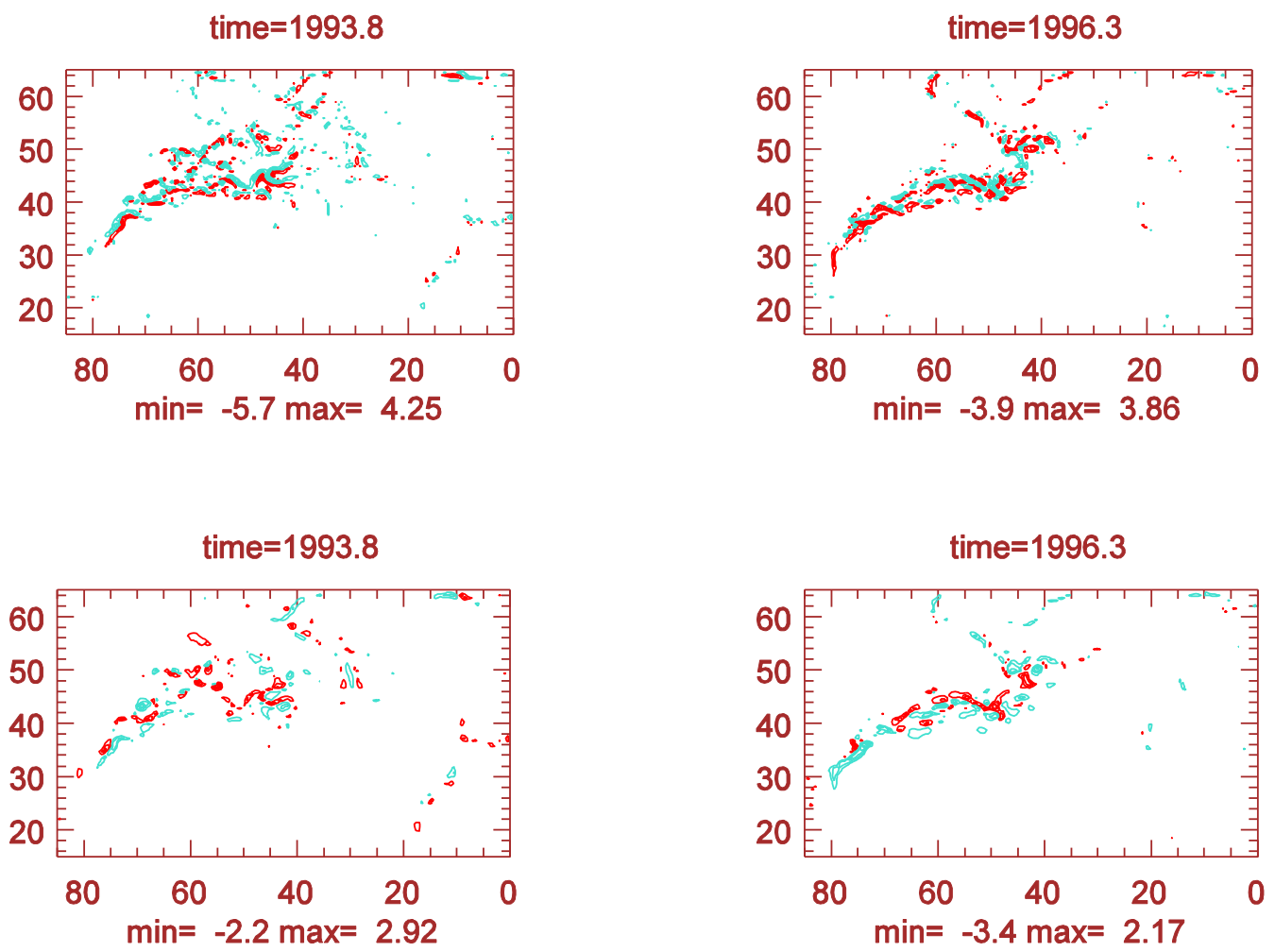

Fig. 3: (a) The mechanism for atmospheric jet generation proposed by Feliks et al. (2004; FGS04 for short): the oceanic jet (heavy blue arrow) gives rise to an atmospheric jet stream (black arrow above), accompanied by a double-gyre circulation (red and blue circles), up to the tropopause level; warm color indicates warm side of the oceanic front. (b) Snapshots of the thermal component $\alpha \nabla^{2} T$ (upper panels) of the vertical velocity $w\left(H_{E}\right)$, and of the mechanical component $\gamma \nabla^{2} \psi$ (lower panels), in the North Atlantic over the area of Fig. 1; red contours indicate upward velocity, i.e., anticyclonic rotation in the horizontal. 

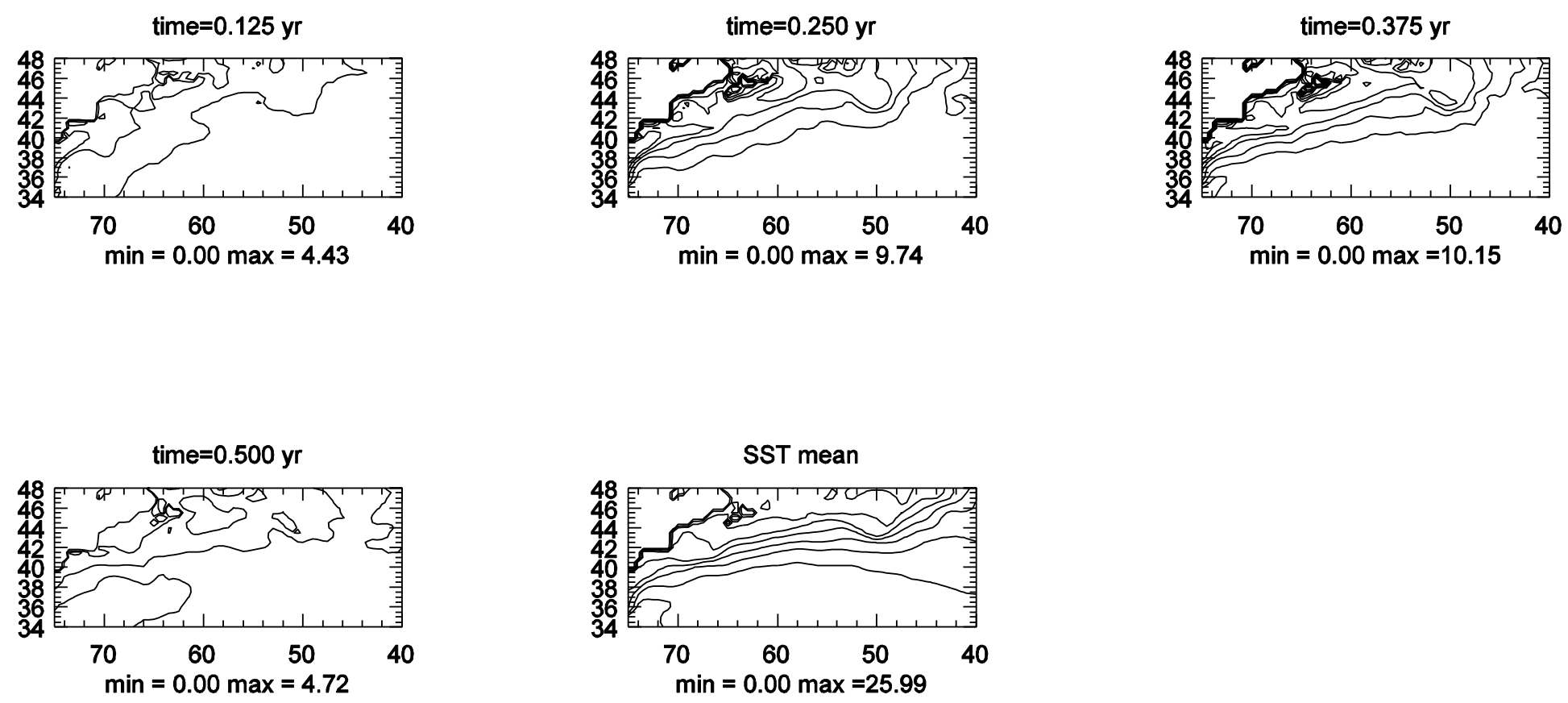

Fig. 4: Seasonal cycle of the SST field in the western North Atlantic, (32 N-48 N, 75 W-40 W), for the 50 years 1958-2007, with the year divided in eighths, rather than months. Composites of the 1-yr mode, in four phase categories, correspond to one half of the annual cycle, namely June-to-November; the phases are labeled by the mid-point of the phase category. The panels look very similar for the other half of the cycle, December-May, only with opposite sign; that is the spatial pattern in the panel for $0.625 \mathrm{yr}$ (not shown) $\approx-$ (panel for $0.125 \mathrm{yr})$, etc. These composites are based on the M-SSA pair $(1,2)$, which captures $90 \%$ of the total variance (positive contours solid, negative ones dashed); $\mathrm{CI}=1 \mathrm{~K}$ for the se four panels, and $\mathrm{CI}=1.7 \mathrm{~K}$ for the mean SST field (last panel). 


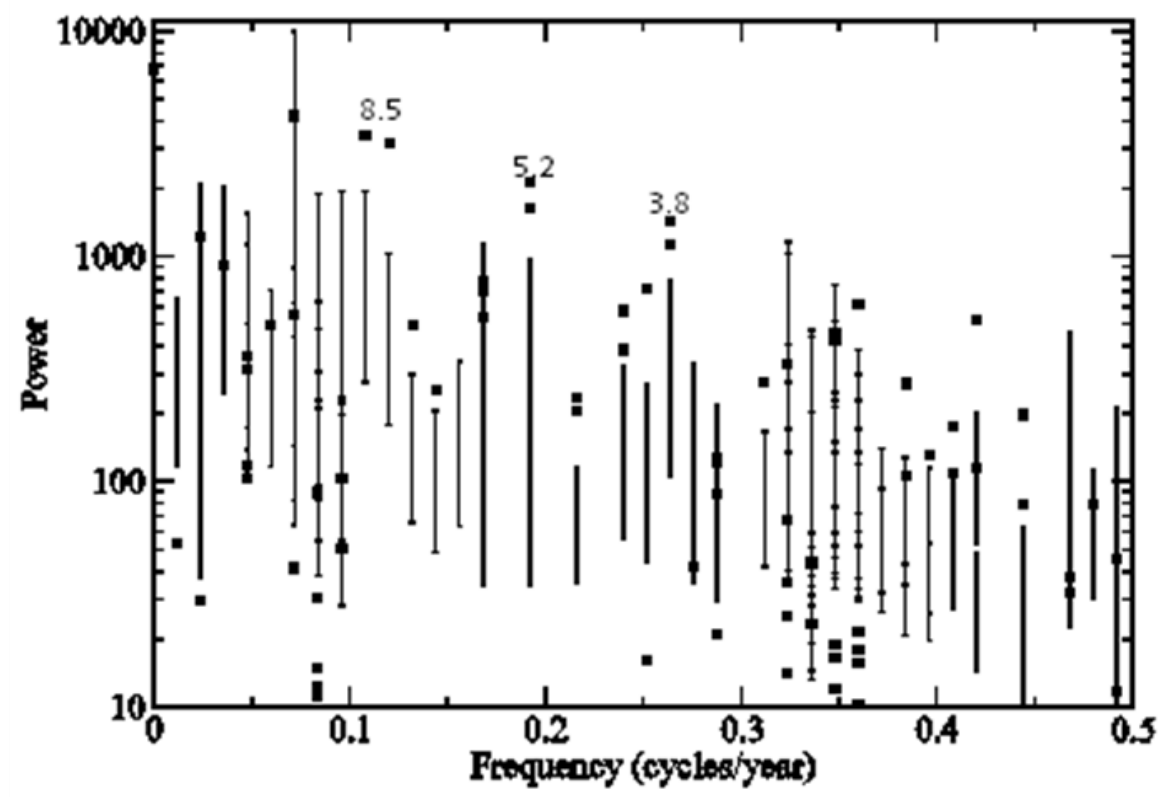

Fig. 5: Spectral analysis of the SST evolution in the CHR region (34 N-43.50 N, $75 \mathrm{~W}-60 \mathrm{~W}$ ), using Monte Carlo MSSA (MC-MSSA) with a window width of $M=150$ months, after having subtracted the seasonal cycle. The estimated variance of each mode in the dataset is shown as a filled black square, while lower and upper ticks on the error bars indicate the 5th and 95th percentiles of a red-noise process constructed from a surrogate data ensemble of 100 time series; each of the surrogates has the same variance and lag-one autocorrelation as the original record. The surrogate time series (Allen and Smith, 1996) were produced by projecting the first 10 principal components of the MSSA analysis onto the basis vectors of a red-noise process. Highly significant pairs are labeled by their period. 


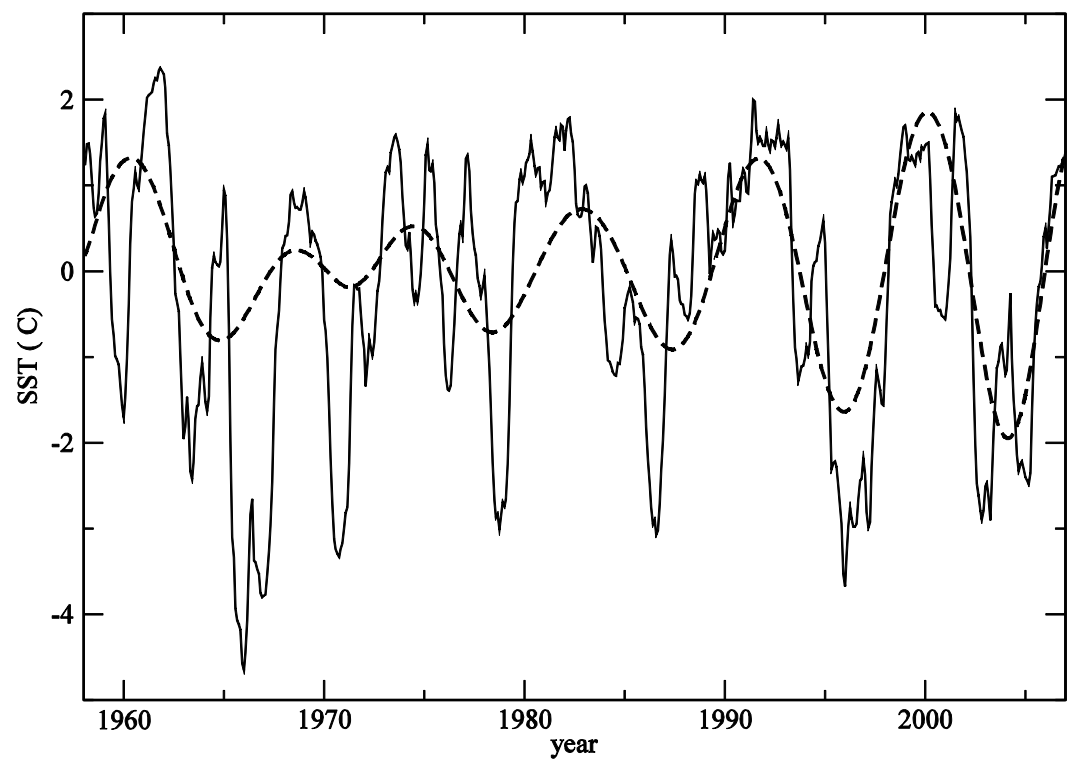

Fig. 6: The evolution of the reconstructed 8.5-yr oscillatory mode in SST, at the point (37 N, 74 $\mathrm{W})$, where its amplitude in the CHR region is maximal; raw data shown as the solid line, and RCs $(3,4)$, which capture the oscillation, as the dashed line. 

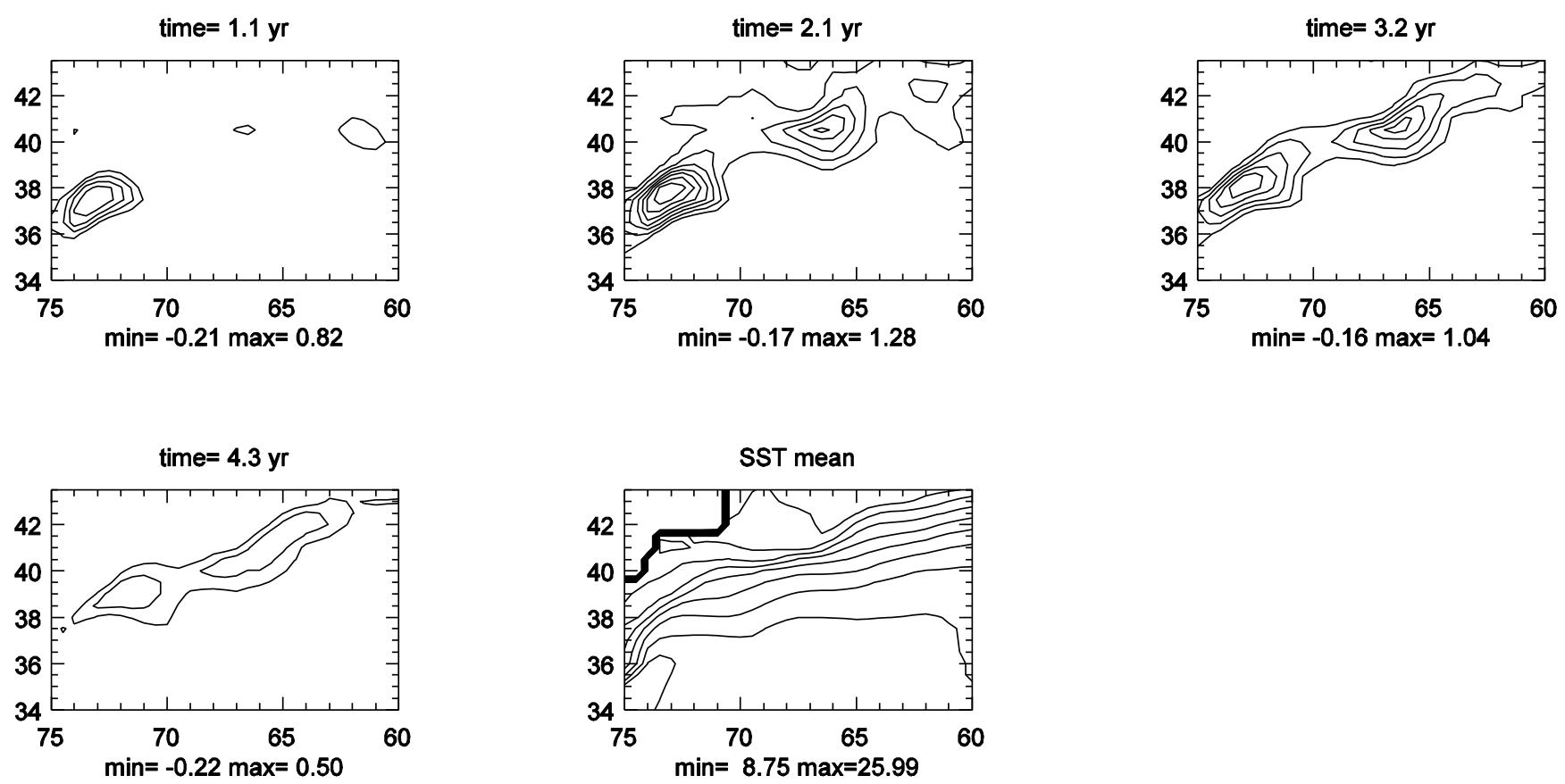

Fig. 7: Composites of the 8.5-yr mode of SST in the CHR region, in four phase categories, which correspond to the first half of the period. These composites are based on the MSSA pair $(3,4)$, which captures $13 \%$ of the total variance (positive contours solid, negative ones dashed). The phase categories are labeled by their mid-point, as in Fig. 4, and CI = 0.15 K; the mean SST (last panel) has CI $=1.7 \mathrm{~K}$. 

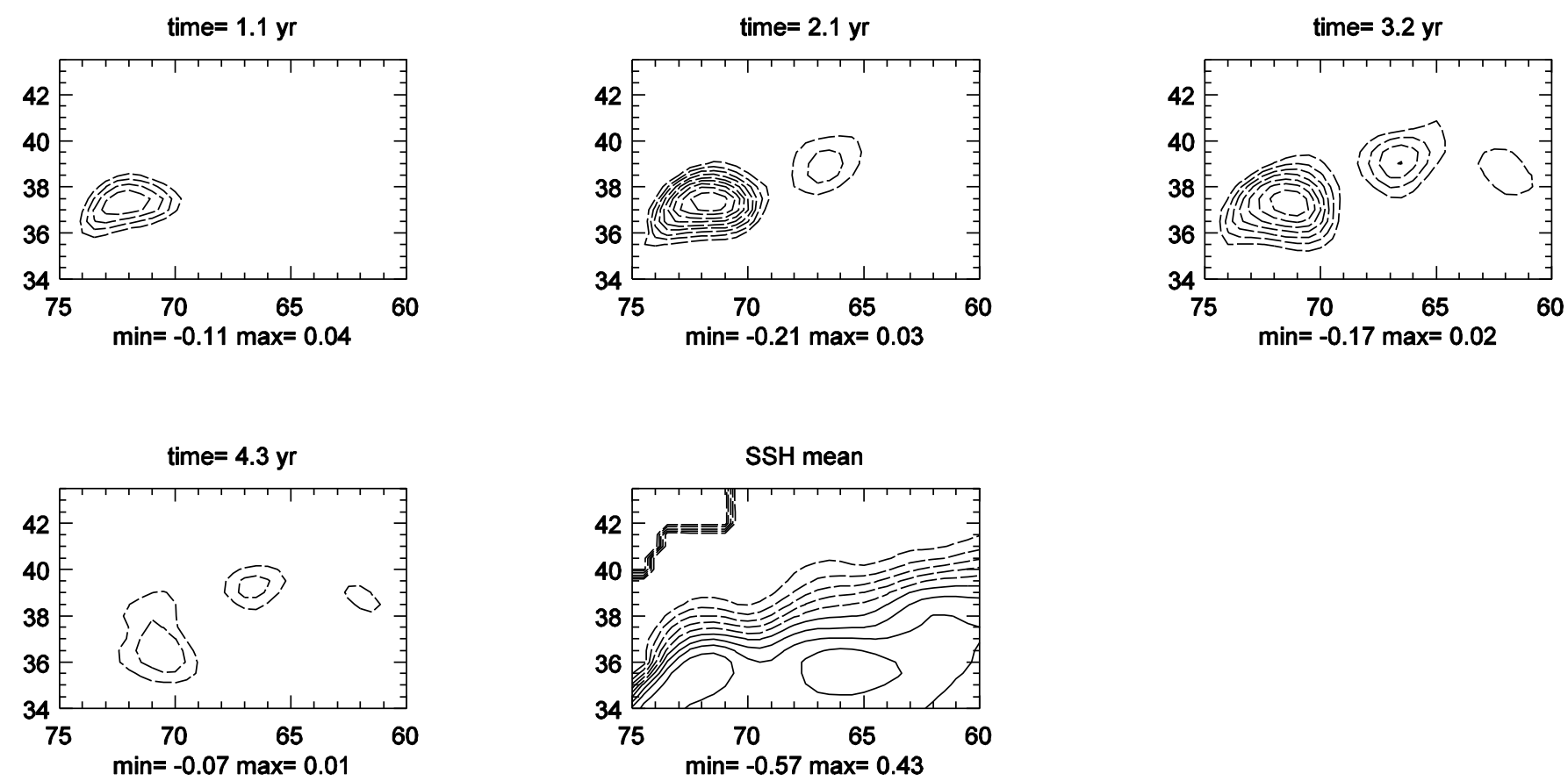

Fig. 8: Composites of the 8.5-yr mode of SSH in the CHR region; same ordering and labeling of panels, and same convention for contours, as in Fig. 7. The four phase composites are based on the M-SSA pair $(3,4)$, which captures $12 \%$ of the total variance; $\mathrm{CI}=0.02 \mathrm{~m}$ here, while CI $=0.13 \mathrm{~m}$ for the mean SSH field (last panel). 


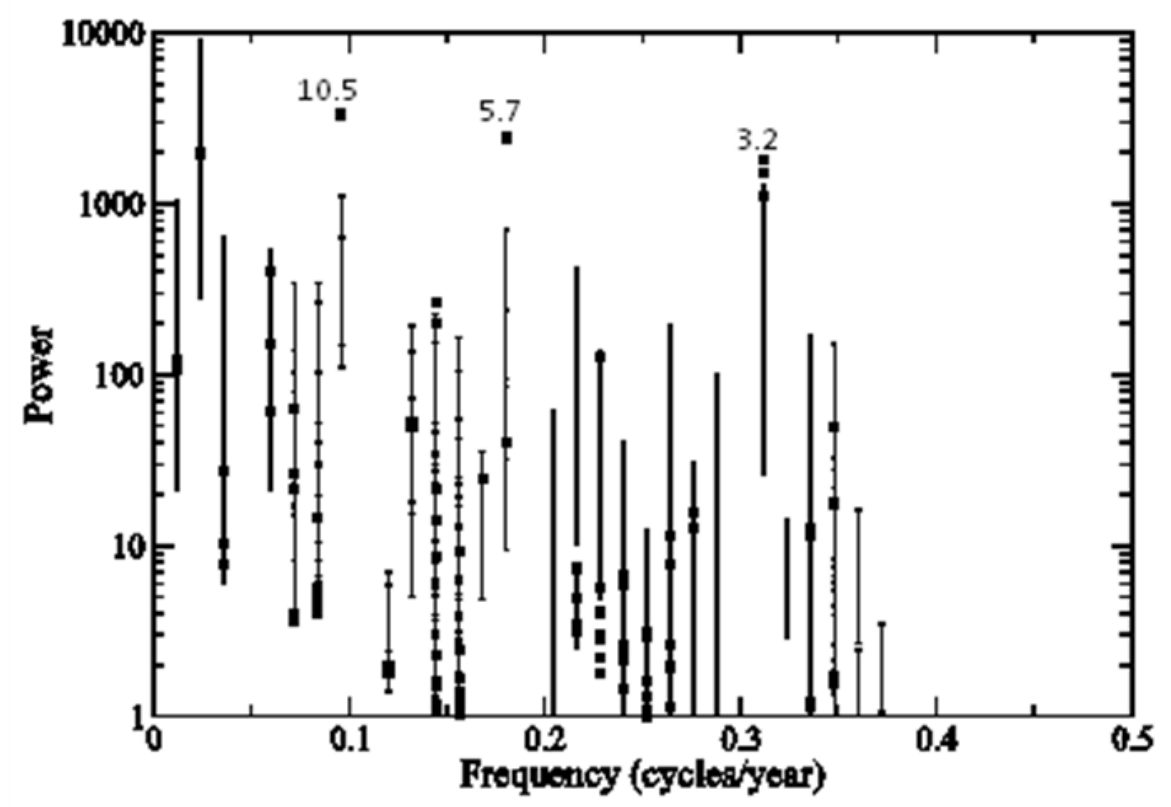

Fig. 9: Spectral analysis of the SST evolution in the GBR region (42 N-50 N, $55 \mathrm{~W}-35 \mathrm{~W}$ ), after detrending and by using MC-MSSA with a window width of $M=150$ months. Same conventions as in Fig. 5; surrogate time series produced the same way as there. 


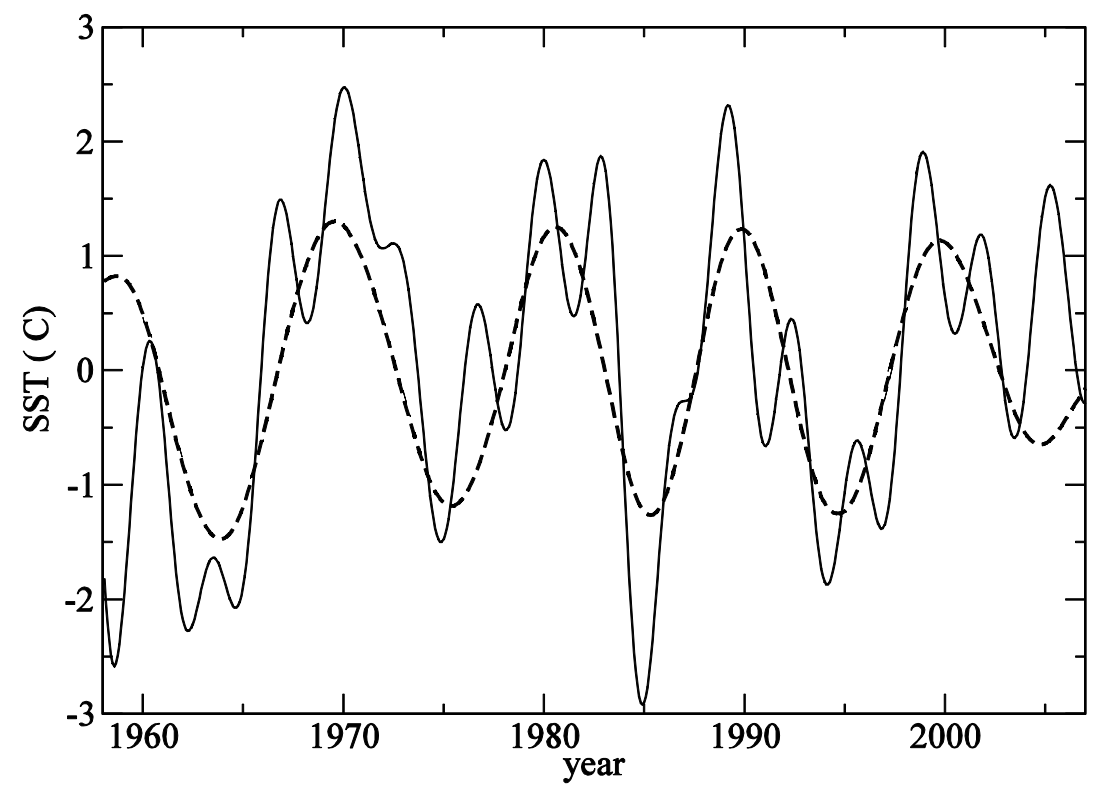

Fig. 10: The evolution of the reconstructed 10.5-yr oscillatory mode in SST, at the point (47 W, $44 \mathrm{~N}$ ), where its amplitude is maximal in the GBR region; raw data shown as the solid line, and RCs $(1,2)$, which capture the oscillation, as the dashed line. 

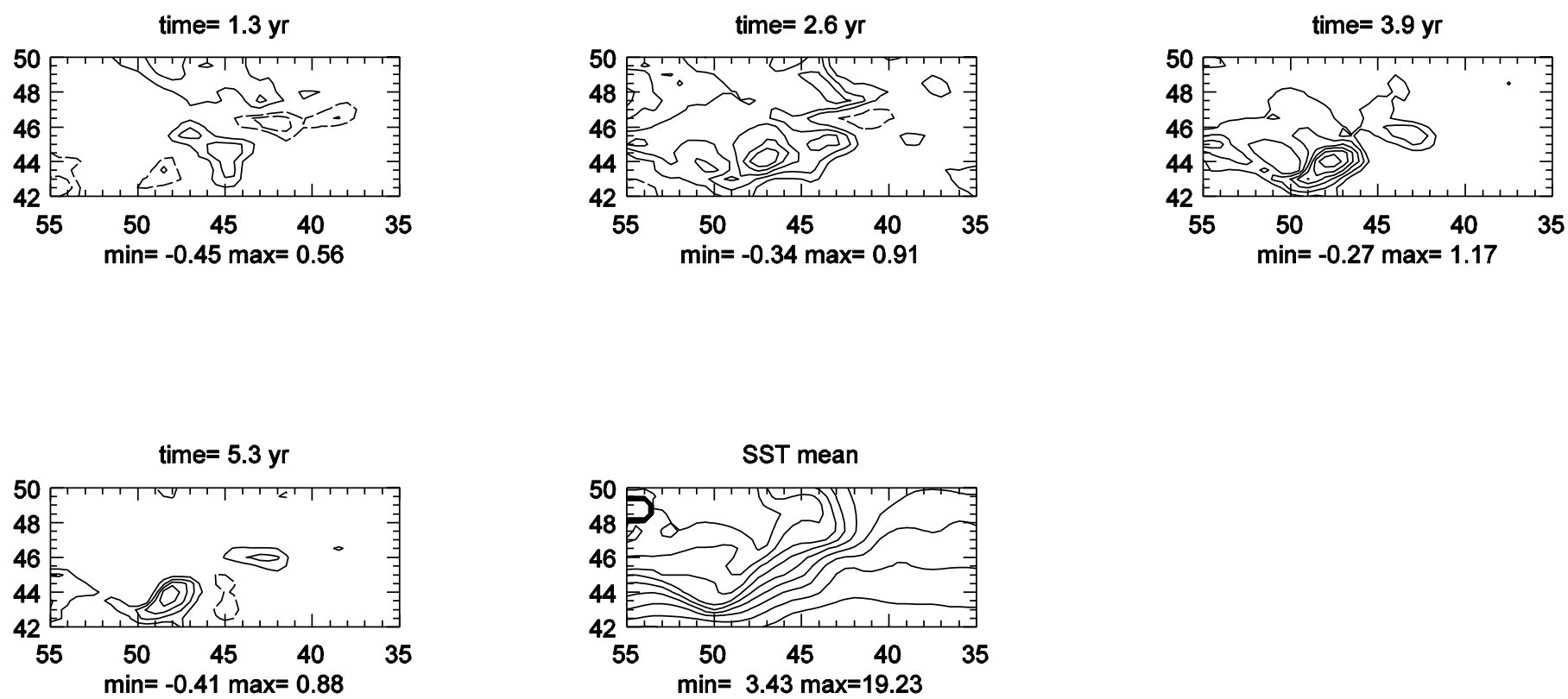

Fig. 11: Composites of the 10.5-yr mode of SST in the GBR region; same ordering and labeling of panels, and same convention for contours, as in Fig. 7. The four phase composites are based on the MSSA pair $(1,2)$, which captures $10 \%$ of the total variance; $\mathrm{CI}=0.15 \mathrm{~K}$ here, while $\mathrm{CI}=$ $1.7 \mathrm{~K}$ for the mean SST field (last panel). 
time $=1993.8$

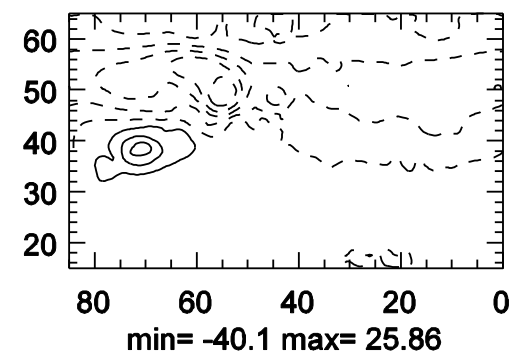

time $=1993.8$

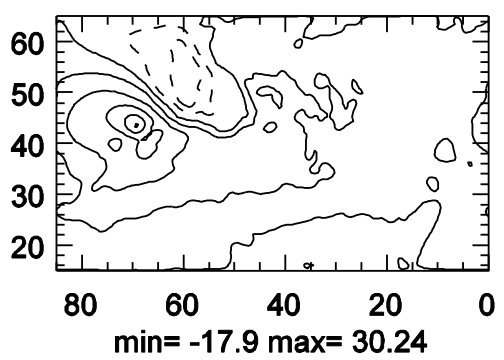

time $=1993.8$

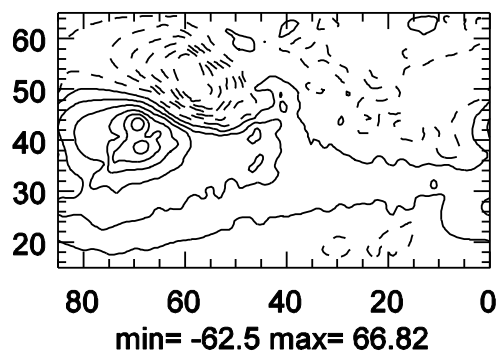

time $=1993.8$

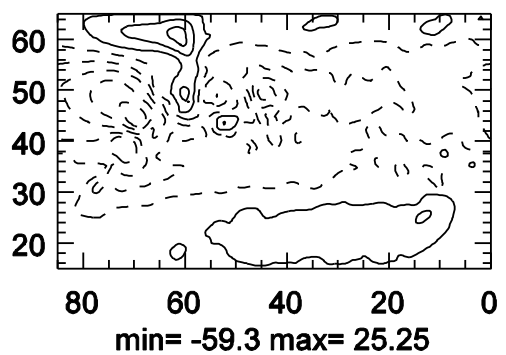

time=1996.3

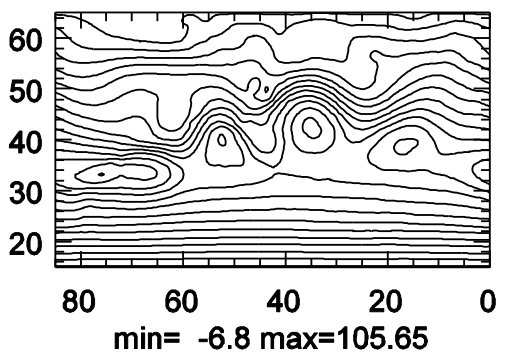

time $=1996.3$

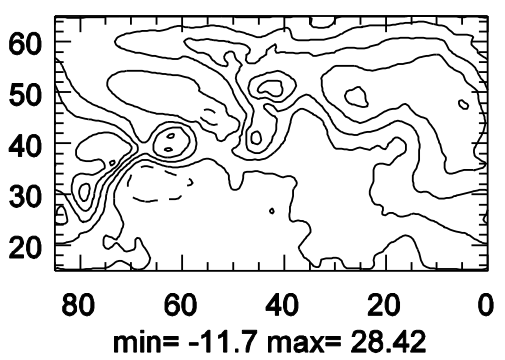

time $=1996.3$

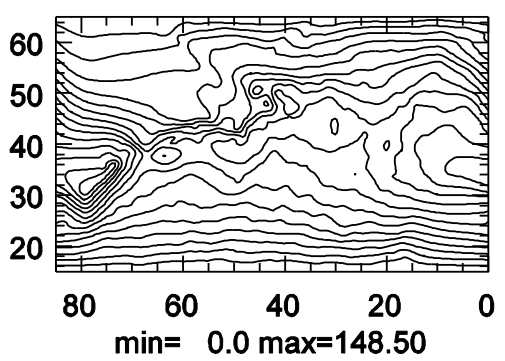

time $=1996.3$

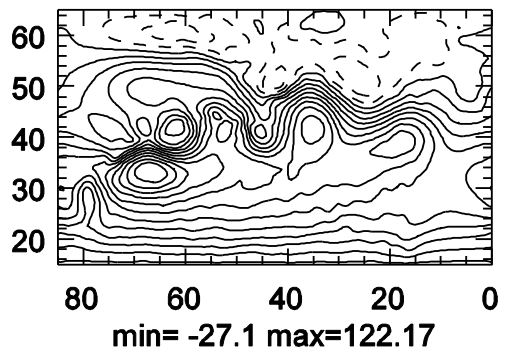

time $=\mathbf{2 0 0 7 . 3}$

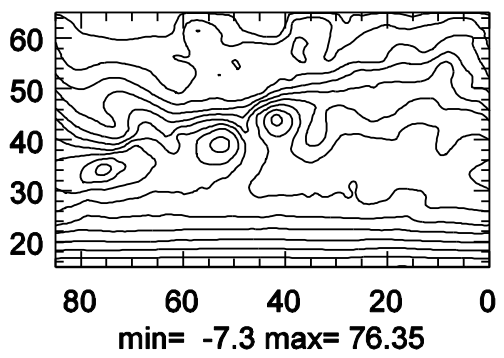

time $=2007.3$

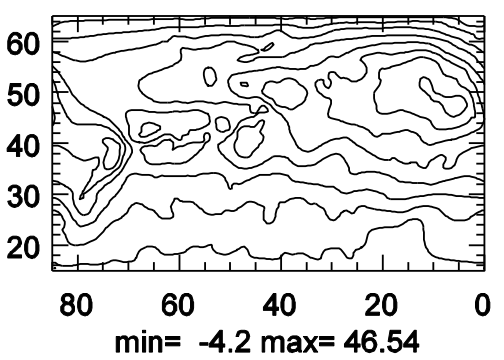

time $=\mathbf{2 0 0 7 . 3}$

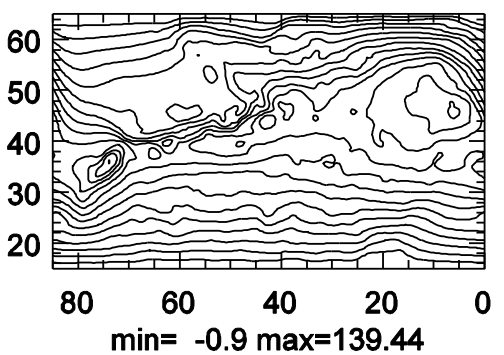

time $=\mathbf{2 0 0 7 . 3}$

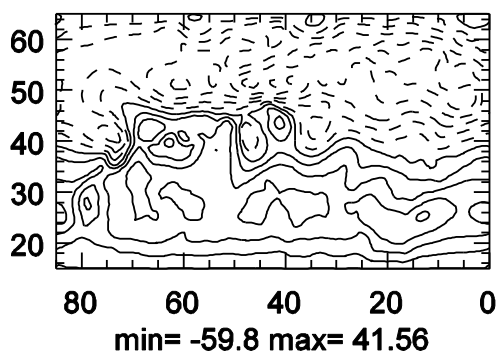

Fig. 12: Evolution of the atmospheric jet induced by the SODA history of the SST field in the coupled MABL-QG model of Feliks et al. (2007; FGS07 for short). Snapshots shown at unequally spaced times, with the time, as well as the maximum and minimum values given in the legend of each plot; positive contours solid, negative contours dashed. First row: barotropic component, $\mathrm{CI}=8$; second row: baroclinic component, $\mathrm{CI}=6$; third row: surface streamfunction, $\mathrm{CI}=10$; bottom row: streamfunction at an altitude of $10 \mathrm{~km}, \mathrm{CI}=10$; nondimensional units. 


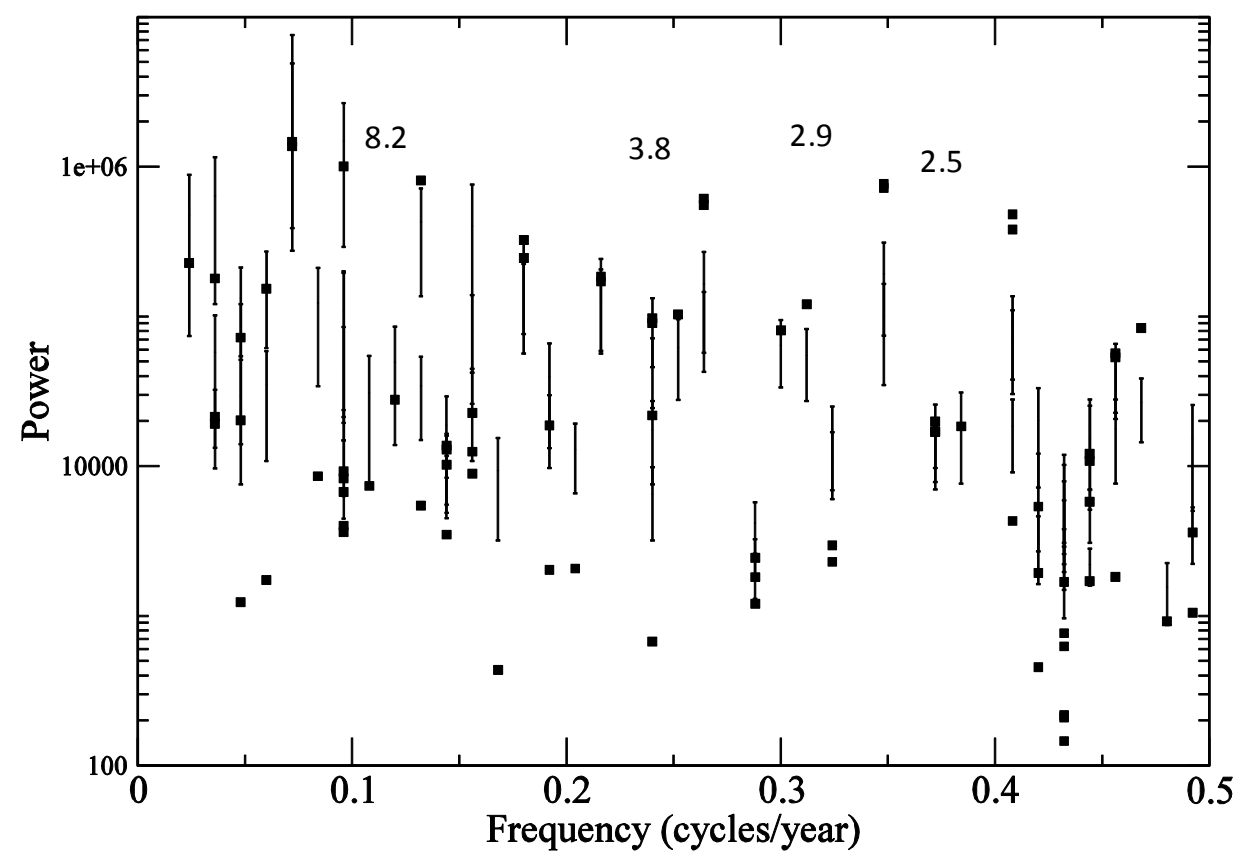

Fig. 13: Spectral analysis of the atmospheric model's combined barotropic and baroclinic modes using MSSA. The MC-MSSA spectrum shown here is computed with a window width of $M=150$ months; other details as in Figs. 5 and 9. 

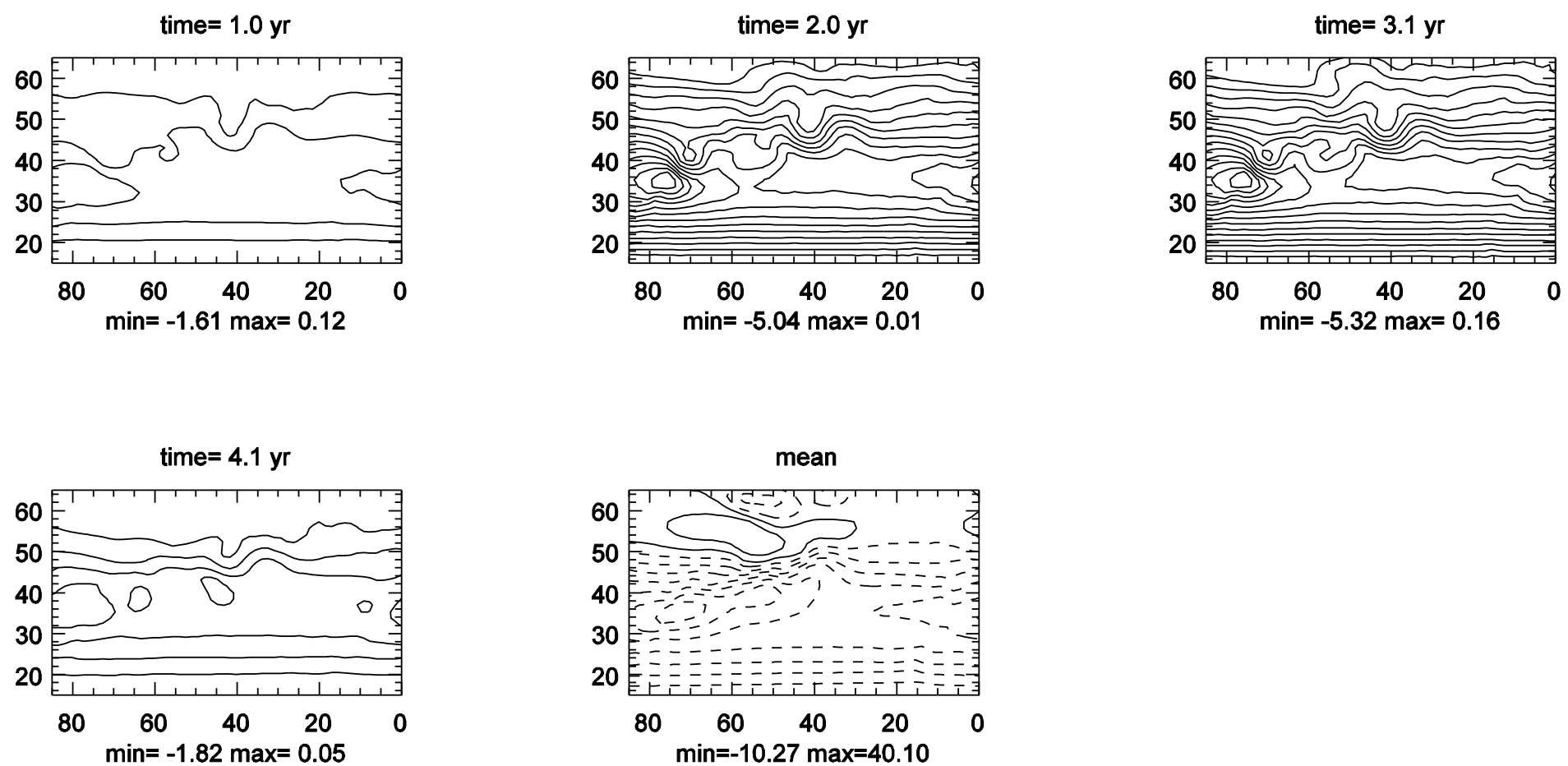

Fig. 14: Composites of the 7.8-10-yr mode of the atmospheric model's barotropic component, in four phase categories, like for the oceanic composites in Figs. 4, 7, 8 and 11. These composites are based on the M-SSA pair $(3,4)$, which captures $15 \%$ of the total variance; here $\mathrm{CI}=0.25$. Note that this broad peak in the atmospheric model encompasses the narrower oceanic peaks at $8.5 \mathrm{yr}$ and $10.5 \mathrm{yr}$ in the CHR and GBR regions, respectively; see Figs. 5 and 9. The mean field (last panel) has $\mathrm{CI}=4.9$ (nondimensional units). 

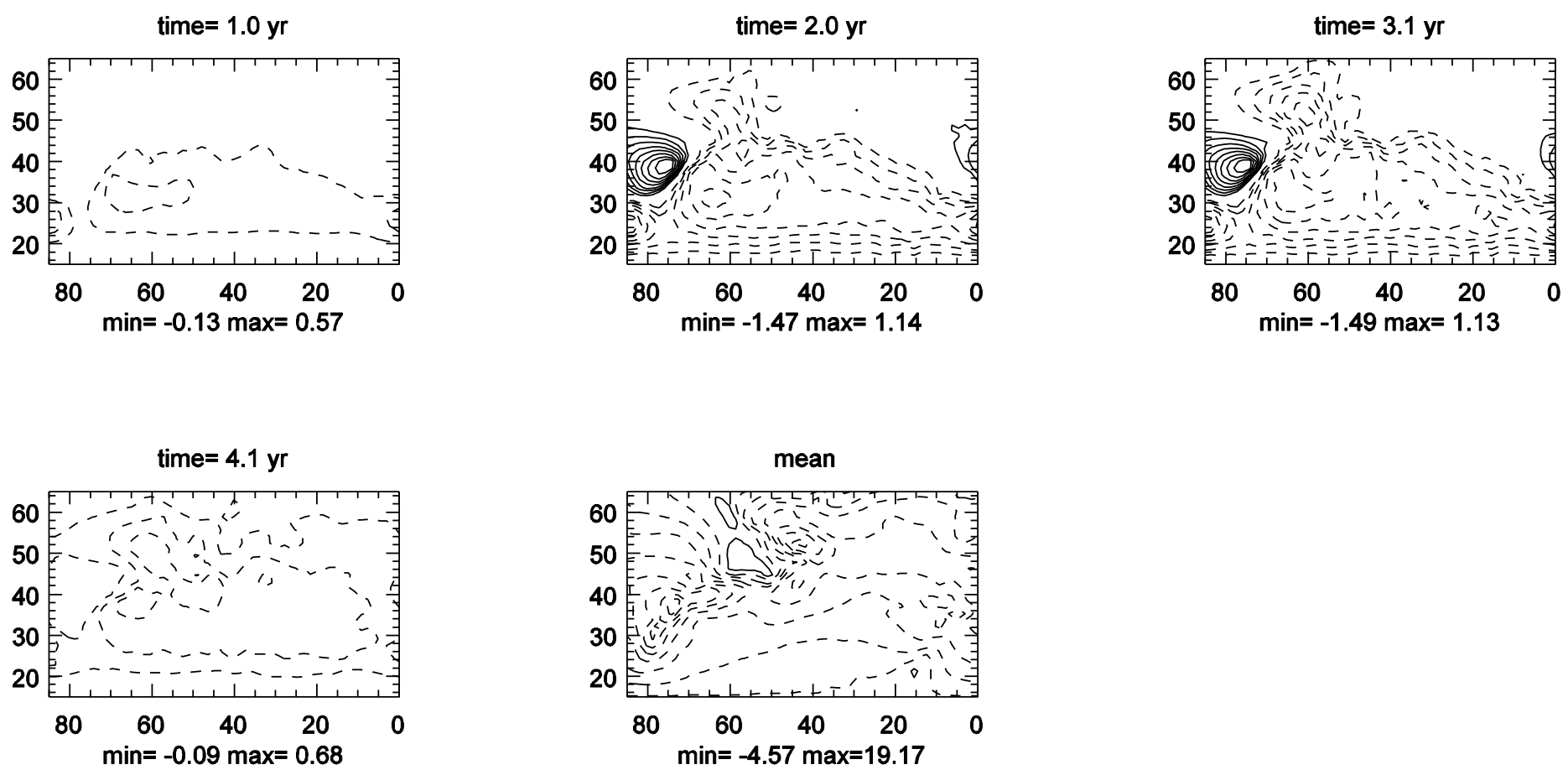

Fig. 15: Same as Fig. 14 but for the baroclinic component; $C I=0.15$ for the four composites and $\mathrm{CI}=2.3$ for the mean. 
(a)

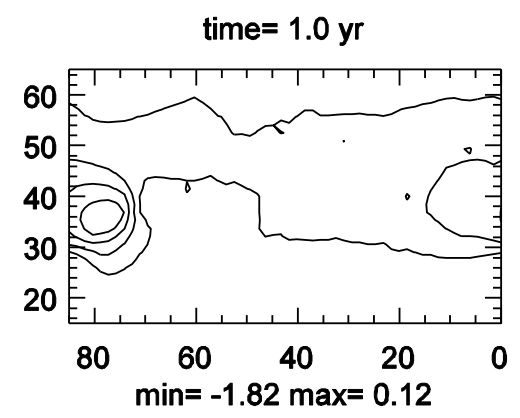

time $=4.1 \mathrm{yr}$

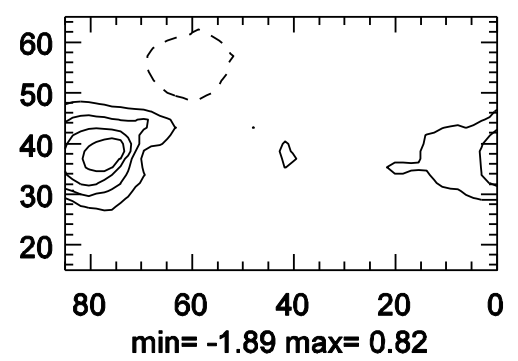

(b) time $=.125 \mathrm{yr}$

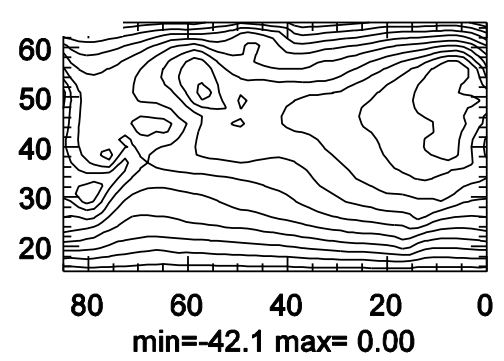

time $=.500 \mathrm{yr}$

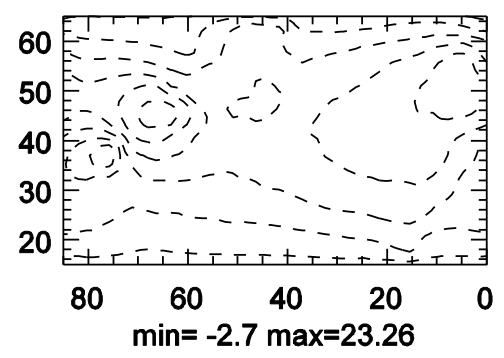

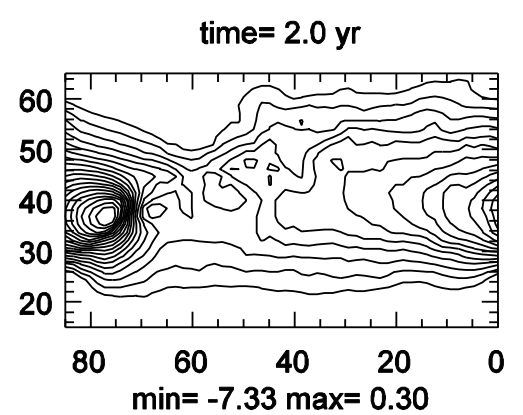
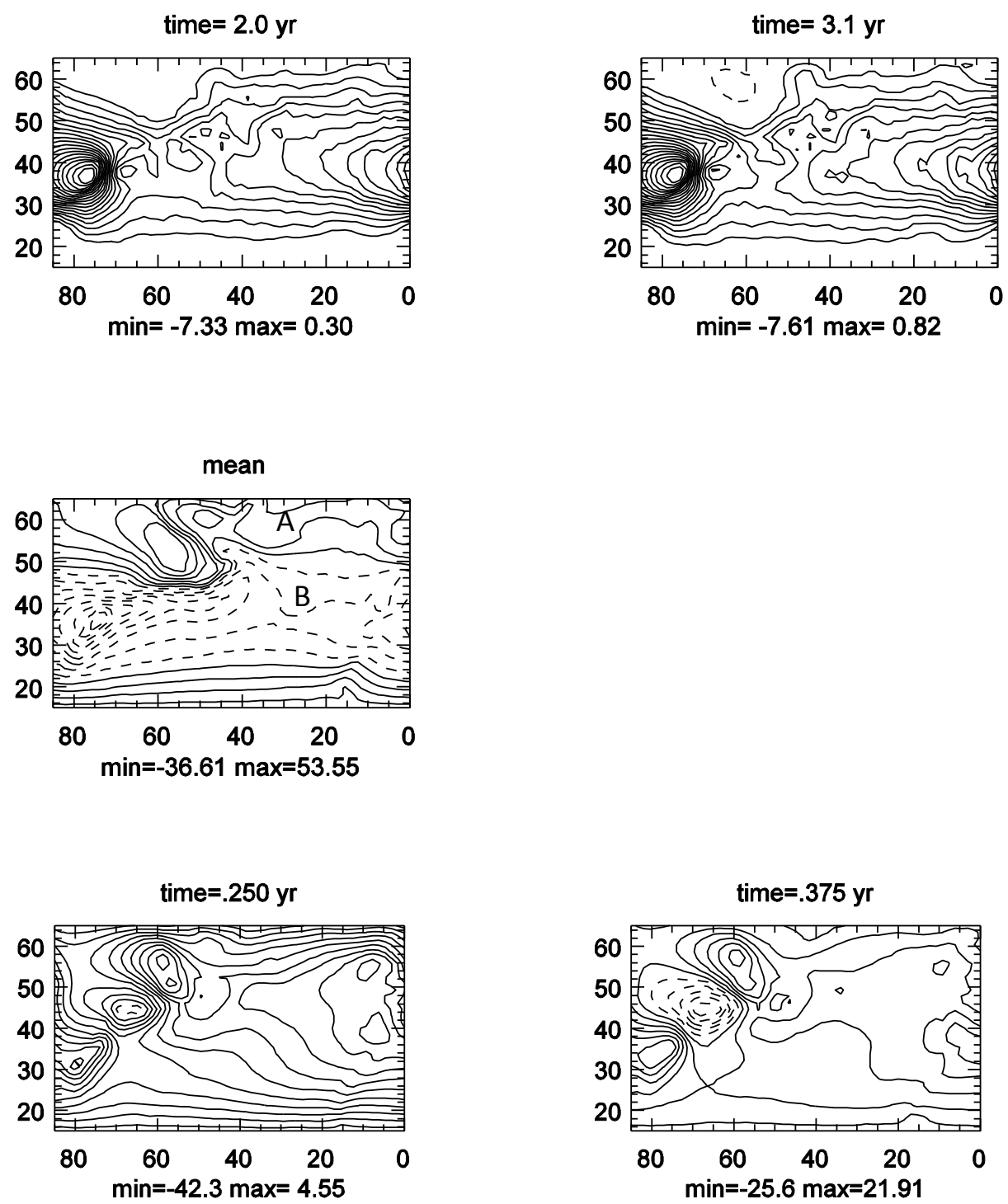

Fig. 16: Streamfunction field near the surface. (a) Composites of the 7.8-10-yr mode, as for Figs. 14 and 15; CI $=0.35$ for the four composites and $\mathrm{CI}=6.0$ for the mean. For greater legibility, the average value of the field over the entire integration domain $(\psi=21)$ has been subtracted from the mean-field panel; this yields an approximately equal number of dashed and solid contours, rather than a preponderance of dashed ones. (b) Composites of the seasonal cycle, i.e. the 1-yr mode, of the atmospheric model, in four phase categories; same phases and conventions as in Fig. 4, for the oceanic composites. These composites are based on the M-SSA pair $(1,2)$, which captures $40 \%$ of the total variance; here $\mathrm{CI}=4$. 

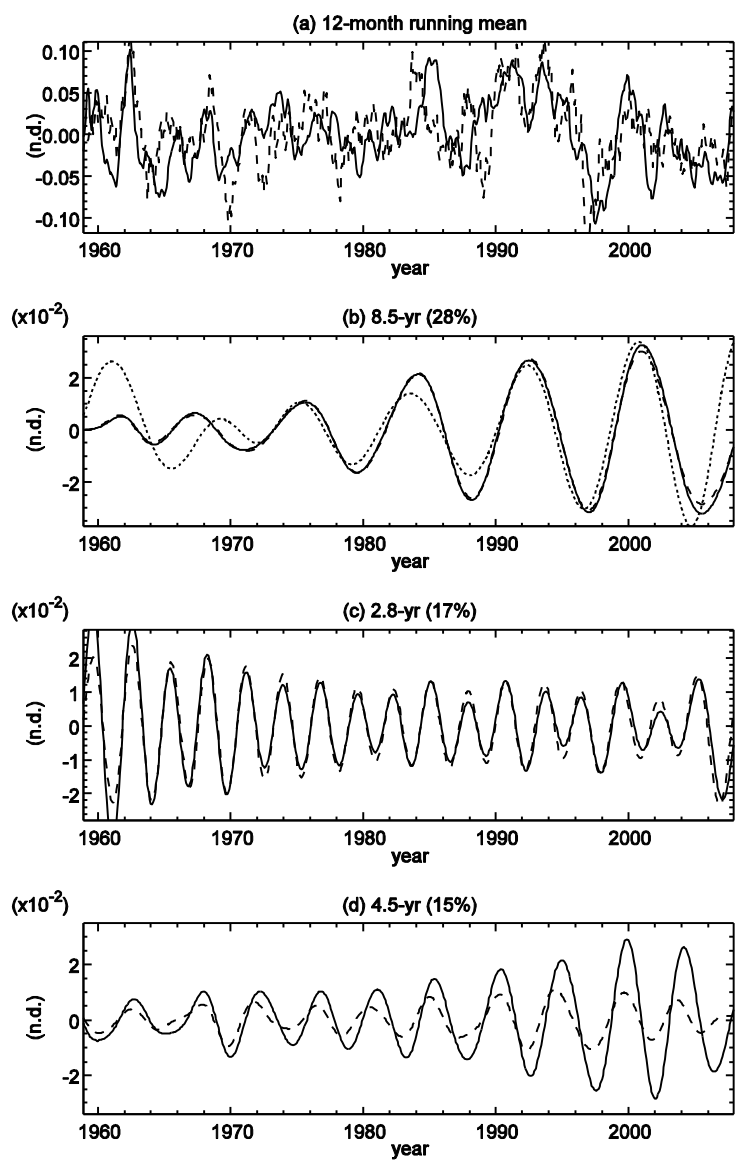

Fig. 17: The observed NAO index (dashed line) and simulated NAO index (SNI; solid line): (a) 12-month running mean; and $(\mathrm{b}-\mathrm{d})$ the RCs of the significant oscillatory modes in both the observed and simulated NAO indices, ordered by decreasing variance, along with the associated periods and variances. The dotted line in panel (b) is the reconstructed 8.5 -yr oscillatory mode in the SST at the point (37 N, $74 \mathrm{~W}$ ) that was shown already in Fig. 6; it is divided here by 45.6 , for proper scaling to the nondimensional RCs. 


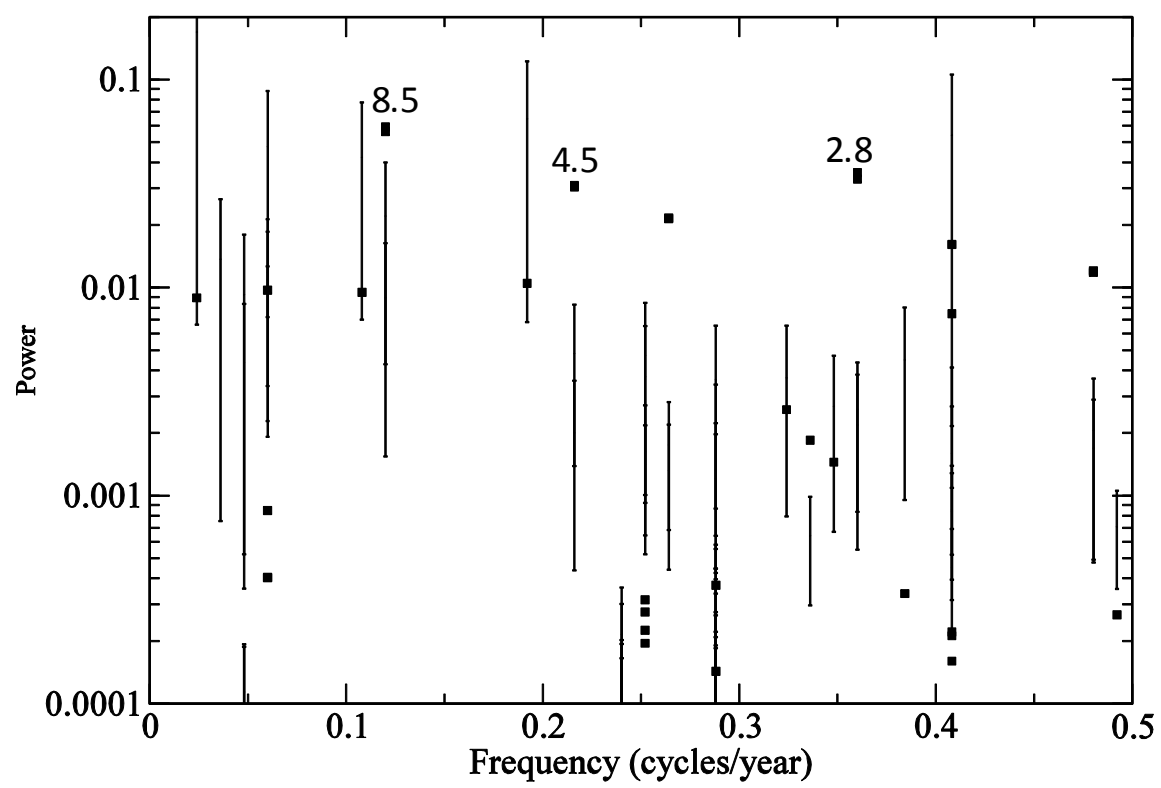

Fig. 18: Spectral analysis of the observed NAO index and the atmospheric model's SNI, obtained by MC-MSSA with a window width of $M=200$ months; other details as in Fig. 9. 


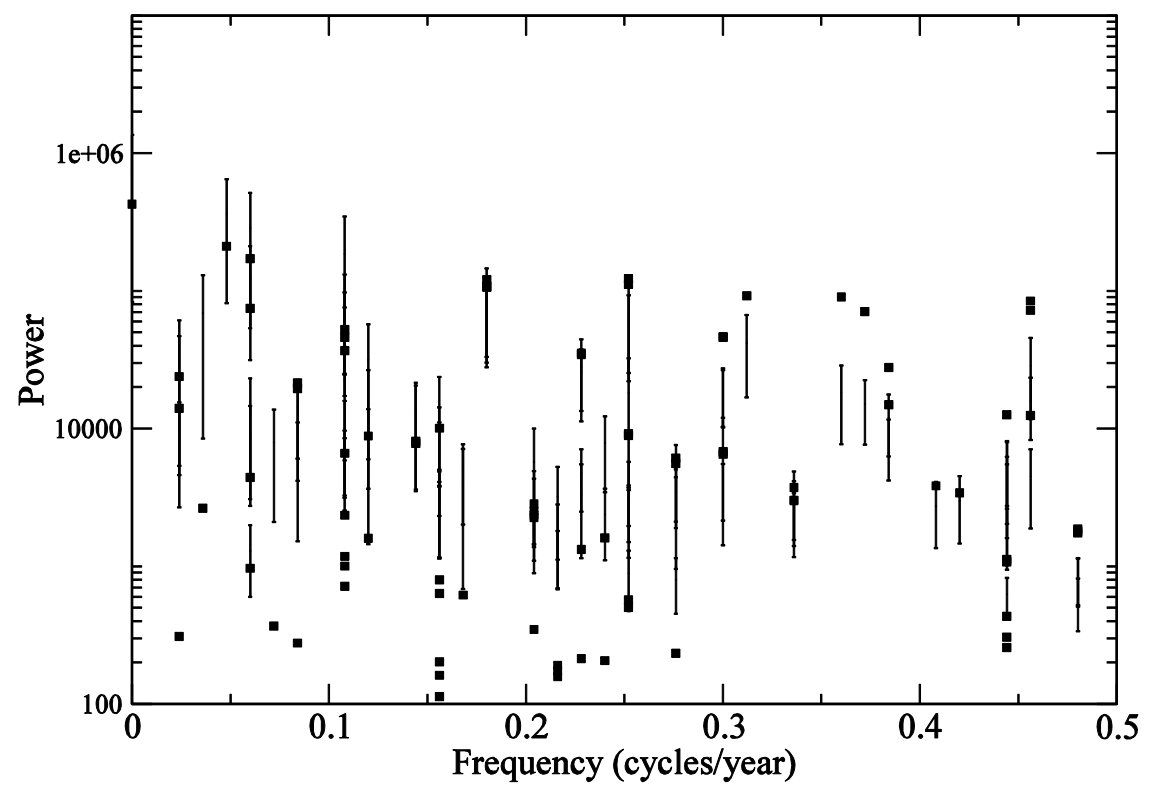

Fig. 19: Same as Fig. 13, but when the atmospheric model is driven by a flat SST field, with $\nabla^{2} T=0$, in the domain (32 N-50 N, $\left.85 \mathrm{~W}-40 \mathrm{~W}\right)$; MC-MSSA spectrum computed with a window width of $M=150$ months, and same conventions as in Figs. 9 and 13. 

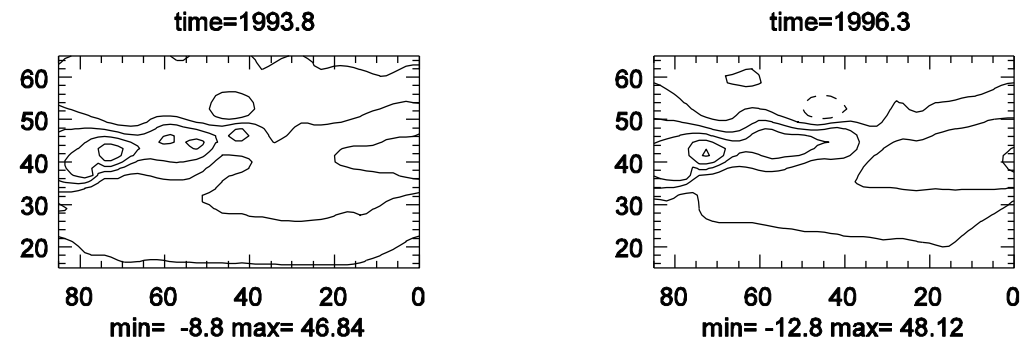

Fig. 20: Evolution of the atmospheric streamfunction at an altitude of $10 \mathrm{~km}$, but when the atmospheric model is driven by a spatially smoothed SST field; see text for details. The two snapshots are shown at the times of weakest and strongest jet, respectively; same CI and other conventions as in the lowermost row of Fig. 12. 


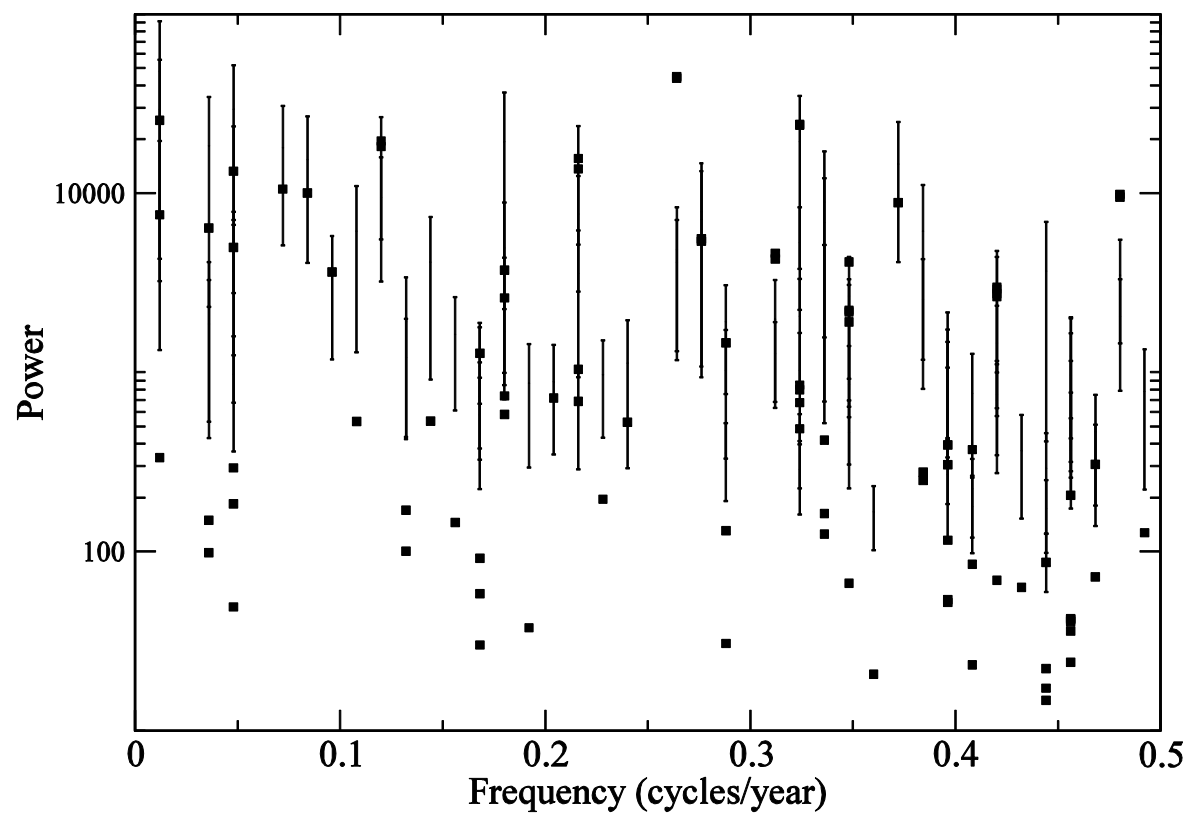

Fig. 21: Spectral analysis of the atmospheric model's combined barotropic and baroclinic modes but when the atmospheric model is driven by a spatially smoothed SST field; see text for details. MC-MSSA spectrum computed with a window width of $M=150$ months, and same conventions as in Figs. 9 and 13. 


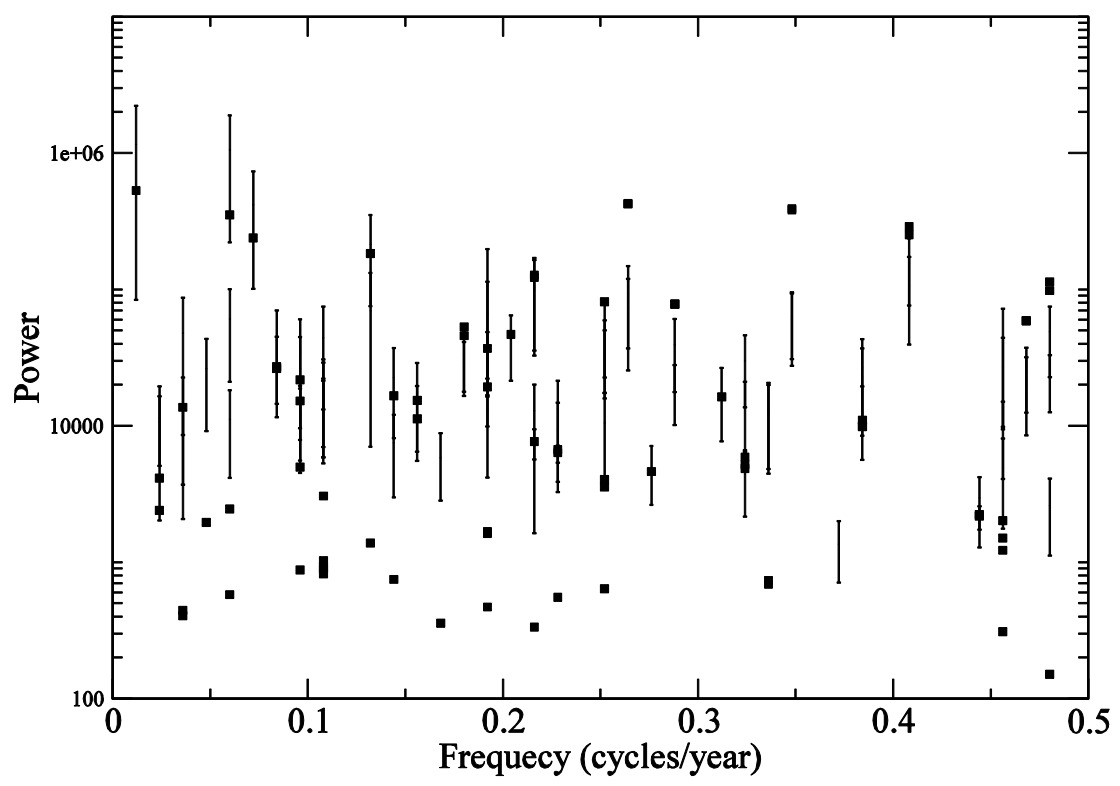

Fig. 22: Spectral analysis of the atmospheric model's combined barotropic and baroclinic modes, as induced by the SST history from SODA but filtering out periods longer than 2 years. MC-MSSA spectrum computed with a window width of $M=150$ months, and same conventions as in Figs. 9 and 13. 